“(C) 2016 IEEE. Personal use of this material is permitted. Permission from IEEE must be obtained for all other uses, in any current or future media, including reprinting/republishing this material for advertising or promotional purposes, creating new collective works, for resale or redistribution to servers or lists, or reuse of any copyrighted component of this work in other works." 


\title{
A Low-Latency Zone-Based Cooperative Spectrum Sensing
}

\author{
Deepak G. C., Student Member, IEEE, and Keivan Navaie, Senior Member, IEEE
}

\begin{abstract}
In this paper we propose a spectrum sensing scheme for wireless systems with low latency requirement such as machine-to-machine communications. In such systems with high spatial density of the base stations and users/objects, spectrum sharing enables spectrum reuse across very small regions. This however needs efficient incorporation of sensors' location information into spectrum sensing. We propose a multi-channel cooperative spectrum sensing technique in which an independent network of sensors, namely monitoring network, detects the spectrum availability. The monitoring network divides the coverage area into overlapped but independent zones. This enables exploiting high spatial distribution without incorporating exact sensors' location. Corresponding to each zone, a zone aggregator (ZA) is introduced which processes the sensors' output. The aggregated decision in each zone associated with the ZA's location is then passed to a decision fusion center (DFC). The secondary base station (SBS) accordingly allocates the available channels to maximize the spectral efficiency. We formulate the function of the DFC as an optimization problem with the objective of maximizing the spectral efficiency. For energy detector sensors, we further obtain optimal detection threshold for different cases with various spatial densities of ZAs and SBSs. This provides extra degrees of freedom in designing the spectrum monitoring network and provides quantitative insight on network design. We further devise an efficient protocol for the proposed technique with very low signaling complexity and show that the proposed method reduces the spectrum sensing latency and results in a higher spectrum efficiency. Extensive simulations confirm our analytical results and indicate a significant improvement in sensing latency and accuracy.
\end{abstract}

Index Terms-Cognitive radio, cooperative spectrum sensing, spectral efficiency, spectrum monitoring.

\section{INTRODUCTION}

Cognitive radio network ( $\mathrm{CRN})$ utilizes dynamic spectrum access (DSA), where the secondary users (SUs) share the radio spectrum with the primary users (PUs). In DSA, SUs should periodically sense the spectrum availability to avoid interfering with the PUs communication activities. In wireless communications, data is often transmitted within time frames. Number of data bits transmitted in each time frame is directly related to the the system throughput. In DSA, part of each time frame is allocated to spectrum sensing thus no transmission is allowed [1], [2]. By increasing the sensing duration the sensing accuracy is also increased however, the remaining time for transmission thus the throughput is decreased. This results in a fundamental trade-off between sensing accuracy and system throughput [3]. As a consequence, choosing the optimal value of sensing duration is a challenging task [4].

The authors are with the School of Computing and Communications, Lancaster University, Lancaster, UK, LA1 4YW, Email: k.navaie@lancaster.ac.uk. The work in this paper is supported by EU Marie Curie CIG 304086-S3iSE. This paper was presented in part at the Intl. Symp. on Wireless Comm. System (ISWCS), Ilmenau, Gernamy, 2013.
Conventionally, the spectrum availability is sensed at the SUs. Fundamental characteristics of multiuser wireless environments including multipath fading, user mobility and hidden terminal problem, as well as limited sensing duration result in reducing sensing accuracy. Therefore, in such environments conventional sensing mechanisms are not able to efficiently sense the spectrum availability with an acceptable level of accuracy required for protecting the PUs [5].

To address the sensing accuracy issue, cooperative spectrum sensing techniques are introduced, see, e.g., [6], [7], [8]. Spectrum availability decision is made by combining the collected sensing information by SUs based on a rule, e.g., AND, OR or K-out-of-N [3]. In such methods, the spectrum availability information obtained from multiple SUs can also be processed using more sophisticated techniques. Instances include weighting [9], multidimensional correlation [10] and minimizing the collision probability at the PUs [5]. In weighting, the share of the provided information by each sensor in the final decision is determined by a weighting vector which is a system design parameter. Further, [10] leverages the spatio-temporal correlations between spectral observations among various nodes and across different time instants to minimize the sensing cost and maximize its accuracy.

Various settings have been proposed for implementing cooperative spectrum sensing, see, e.g., [11] and references therein. Cooperative spectrum sensing proposed in [12] divides the coverage area into clusters, where the SUs perform spectrum sensing and base station acts as decision fusion center. The users considered as the cluster heads then make spectrum availability decisions. In such a cooperative sensing model, a higher sensing duration results in a shorter data transmission duration which results degradation in achievable data rate. In addition, the signaling overhead is also higher in the secondary system and the performance is highly sensitive to the reporting channel condition.

The logical cluster formation proposed in [13] is designed to tackle the issues due to the imperfect reporting channel conditions. In [14], the cluster formation is proposed based on the heterogeneous characteristics of PUs and SUs such that users in the same cluster sense the identical set of channels to increase the sensing accuracy. The cluster heads however act locally therefore unable to incorporate their location information into the network wide channel allocation. In addition, various decentralized cooperative schemes are proposed, e.g., [15], where no fusion center exists and therefore the SUs themselves diffuse the received decisions.

In addition to the centralized and decentralized cooperative schemes, a relay-based multiple hops cooperative sensing is proposed in [16], where source to destination spectrum information is forwarded by the relay nodes, where either amplify-and-forward or decode-and-forward is implemented. 
This tackles the issues of erroneous report channel by increasing the cooperation footprint.

As a matter of fact, whether it is centralized, decentralized or relay-assisted cooperative model mentioned in [6]- [16], the formation of clusters is very challenging due to the time varying nature of the wireless channel, and user's mobility. Merits of incorporating the location information are recognized in conventional cognitive radio [17] as well as in advance cooperative communication [11]. However, embedding the location information in the CRN design might increase the signaling overhead. The dynamic cluster formation algorithm also causes very high signaling overhead. Therefore, an independent spectrum monitoring network has been proposed in this paper to improve the cooperative sensing efficiency with reduced complexity.

A two channel sensing technique under imperfect spectrum sensing based on an access, and a backup channel is also proposed in [18], where both channels are sensed in a single time slot to improve the system performance by jointly considering spectrum sensing and spectrum access. Although cooperative sensing often improves the sensing accuracy, the corresponding signaling overhead further reduces the overall system throughput.

In the previously proposed cluster based cooperative sensing approaches, in addition to the signaling overhead due to the cluster head selection, cooperative spectrum sensing also introduces extra spectrum sensing latency. This is due to the fact that the SUs need to allocate an extra part of their fixed time frame to transmit the sensing information to a fusion center and then wait for the sensing decision to be made and received back. To address this issue, the sensor selection algorithms have been proposed in [19], [20]. However, cooperative sensing fails to provide required low-latency access which is of an immense importance in uses-cases including machine-tomachine (M2M) communications [21]. M2M plays an important role in the structure of the Internet-of-Things (IoT) which will be mainly connected through wireless communications.

To tackle the latency issues due to the sensing duration, [22] proposes offloading the cooperative sensing to an independent monitoring network. It comprises of sensors deployed in the coverage area and continuously monitor the spectrum availability. The sensing information is then communicated by the sensors to a central entity on separate signaling channels. In this setting, by careful design of system parameters, the same level of accuracy is achieved without reducing the system throughput. There is, of course, cost associated with building the monitoring network, which is justified in [22] considering extraordinary price of radio spectrum in mobile communication bands. An independent network of sensors is further considered in [23], [24] for nomadic cognitive networks in urban and sub-urban areas. The advantages of considering a separate monitoring network are twofold. Firstly, it lowers the corresponding sensing latency due to the reduced sensing duration, thus the spectral efficiency is increased by offloading the spectrum sensing task to an independent monitoring network. Secondly, the spectrum sensing accuracy is significantly improved due to cooperative sensing.

The above mentioned techniques improve the sensing ac- curacy and its latency but simply ignore the sensors' location information. Due to very high number of objects in the coverage area, incorporating the location information into sensing is capable of enabling spectrum reuse across very small regions in the network coverage area. In this paper we refer to this as micro-spectrum-reuse. Incorporating the exact location of the sensors however might introduce a new dimension to the spectrum sensing complexity and increases its associated costs. Instead in this paper we propose a simple Zone-Based Cooperative Spectrum Sensing. The sensing architecture in the proposed method is based on dividing the coverage area into zones and defining a zone aggregator (ZA) as an intermediate entity. We consider a general case in which the spectrum is divided into number of channels (e.g., sub-channels in multicarrier systems). The ZAs then process the sensing outcome of the sensors for each channel located in their corresponding zone. The aggregated decision for each zone is then passed to a fusion center. In our proposed scheme, to address the overhead issue we further devise a one-bit-per-channel signaling scheme between the ZAs and the fusion center.

In our proposed method a central decision fusion center (DFC) located, e.g., in the secondary base station (SBS) then utilizes the aggregated sensing information in the network zones. SBS accordingly allocates the available channels to maximize the spectral efficiency and keep the interference at the PUs below the system required threshold. We formulate the corresponding function of the DFC as an optimization problem and show that it is a convex optimization problem. We then obtain optimal detection threshold for different cases with various spatial densities of ZAs and SBSs. We further obtain a close form for the optimal sensing threshold based on a weight-based approach.

Various factors are involved in the efficiency of the proposed method in this paper, including number of zones and base stations, the spatial distribution of the sensing devices and the zone size. We investigate the impact of these factors on the system performance and propose techniques for efficient design of the corresponding parameters. This provides extra degrees of freedom in designing the spectrum monitoring network and provides quantitative insight on deployment of such networks. In our analysis, we focus on energy detection as the main spectrum sensing method at the sensors. The analysis presented here can be extended to design the parameters for cases where other spectrum sensing techniques are utilized in the sensors.

In the proposed method, the latency associated with the spectrum sensing is the time required for signaling between the SUs and the DFC. For a given required spectrum sensing accuracy, we also show that the the proposed method in this paper provides a lower latency in comparison with conventional sensing methods ${ }^{1}$. Therefore, the proposed method provides enabling techniques and protocols for adopting DSA in low latency M2M communications.

The analysis presented in this paper are unique as they provide quantitative insight on the achievable gain on the

\footnotetext{
${ }^{1}$ Hereafter, conventional sensing is referred to any spectrum sensing technique in SUs in which the time frames are divided into sensing, and transmission durations.
} 
spectral efficiency using cooperative sensing based on an independent monitoring network.

Using simulations we investigate the accuracy of spectrum sensing in the proposed method as a function of distributed sensing information. The achieved throughput gain of the proposed method for various network parameters, e.g., sensing duration, detection threshold, primary activities, is also investigated. In addition, the proposed zone-based cooperative spectrum sensing method is compared against the reference model where there is no cooperation among the clusters or SBS. Comparisons are also made with the cases where the spectrum sensing information is combined using only OR/AND method.

In the following we summarize the contributions presented in this paper:

1) We propose a novel spectrum sensing method based on an independent spectrum monitoring network and devise the associated system, algorithms and signaling protocols which incorporate zone location information in the spectrum sensing. The proposed method in this paper enables micro-spectrum-reuse and results in higher spectral efficiency, lower signaling overhead, and thus the lower latency.

2) An analytical framework is developed with the objective of maximizing system throughput under various monitoring network scenarios subject to spectrum sensing accuracy and maximum tolerable imposed interference at the PUs.

3) Extensive simulations confirm our analytical results and indicate the throughput performance and sensing latency improvement using the proposed sensing method. The simulation results also outline the parameter design explain the role of various factors including spatial density of ZAs, and SBSs, primary system activity, and sensing threshold on the sensing performance.

The rest of the paper is organized as follows: System model is presented in Section II. The zone-based cooperative spectrum sensing technique, and its performance analysis are presented in Sections III, and Section IV, respectively. Section $\mathrm{V}$ describes the extensive simulation results, which is followed by conclusions in Section VI.

\section{System ModeL}

A schematic of the system is shown in Fig. 1 in which a primary base station (PBS) provides service to the PUs which are randomly distributed within the coverage area. The secondary system is also a cellular network which utilizes orthogonal frequency division multiplexing (OFDM), where the frequency spectrum is divided into $N$ non-overlapping channels. Due to the small-scale frequency-dependent multi-path propagation characteristics, each SU may experience different channel gains across different sub-channels, indexed by $i=1, \ldots, N$, each with bandwidth of $B_{i} \mathrm{~Hz}$. The same spectrum is also utilized by the primary system in the downlink. Depending on the PU activity and its required quality of service (QoS) at a specific time and location, SUs may have access to $M$ channels, where $0 \leq M \leq N$. Without loss of generality,

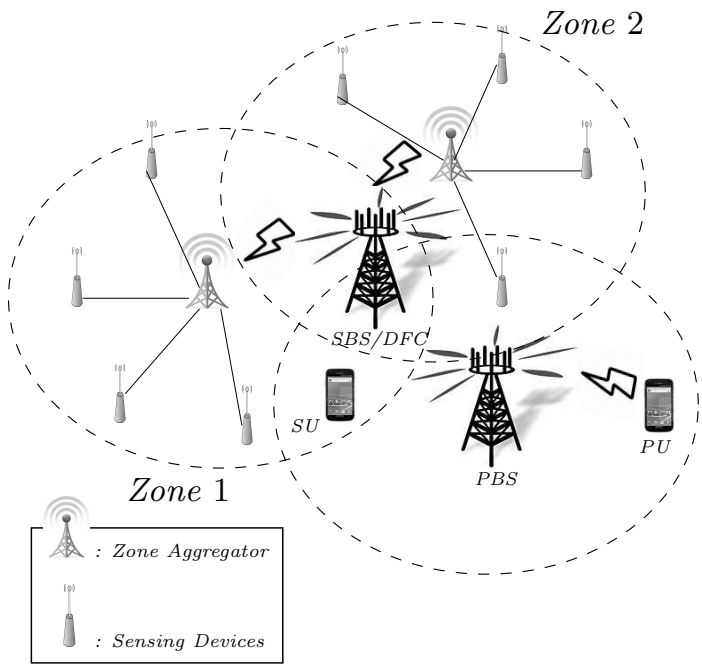

Fig. 1. The zone-based cooperative spectrum sensing.

we also assume that all the base stations are equipped with a single omnidirectional antenna. The analysis can be easily extended into sectorized cells by considering each sector as a cell with a single antenna.

\section{A. Spectrum Monitoring Network}

The spectrum sensors are distributed uniformly within the coverage area. In practice, their location can be engineered by the service providers. For simplicity, we further assume a homogenous network of sensors, where sensing parameters of all the sensor nodes are the same. Unlike the conventional sensing methods, where SUs sense the channel sequentially before accessing them, in the proposed method, the sensing task is offloaded to a spectrum monitoring network. In this setting, each sensing device detects the primary spectrum activity on a subset of channels, $i \in\{1, \ldots, N\}$, within a circular region with radius, $r_{\text {sen }}$ and reports their availability to the SBS. As a result of the proposed independent sensing network, the sensing order of multiple channels becomes irrelevant due to the sufficiently longer sensing duration available. During transmitting the channel availability reports to the zone aggregators, the sensing function is stopped. The connectivity of the sensing network therefore depends on $r_{\text {sen }}$ and distribution of sensing devices.

To associate the sensing information with the location, we then divide the coverage area into overlapped zones. The zones are chosen assuming a uniform distribution of sensing devices. In each zone, there is a zone aggregator (ZA) which receives the sensing information from sensors located in its circular sensing zone with radius $r_{Z A}$. The sensing devices and ZAs collectively form a monitoring network which is designed for cooperative spectrum sensing in the secondary network. Each ZA is associated to the location of its covered zone and broadcast a pilot signal including a zone identification (ZID). Monitoring network utilizes a narrow band pre-allocated spectrum independent from the primary and secondary systems.

The received information in the ZAs is then processed and forwarded to a decision fusion center (DFC) located, e.g., 
in the SBS indexed by $s=1, \ldots, S$. Based on the sensing information provided by the corresponding ZAs, DFC then decides the availability of each channels in that particular zone. Here, ZAs are indexed by $z=1, \ldots, Z$, where $Z$ is the number of zone aggregators in the system.

\section{B. Sensing Devices}

Sensors utilize energy detection technique for detecting the availability of the channels. Energy sensing has been considered here due to its simplicity and tractability as it does not need a priori channel information, see, e.g., [25], and [26].

The sampled signals received at the sensor during the sensing duration are $y_{i}(k)=w_{i}(k)$, and $y_{i}(k)=g_{i}(k) x_{i}(k)+$ $w_{i}(k)$, under hypothesis $H_{0}$ and $H_{1}$, respectively, where $H_{0}\left(H_{1}\right)$ represents the absence (presence) of the primary signals. In addition, $y_{i}(k)$ is the $k$-th received sample over channel $i$ and $g_{i}(k)$ is the channel gain which is assumed to be constant during the signaling duration. Noise signal, $w_{i}(k)$, is assumed to be independent and identically distributed circularly symmetric complex Gaussian with zero mean and variance of $\mathrm{E}\left[\left|w_{i}(k)\right|^{2}\right]=\sigma_{w}^{2}$.

Time is slotted into frames in which the frame duration and the sensing duration for each sensing device are denoted by $T$, and $T_{s, i}$, respectively. The sampling frequency is $f_{s}$, thus the number of samples during the sensing duration is $K=T_{s, i} f_{s}$. The received signal energy is $E_{i}(y)=\frac{1}{K} \sum_{k=1}^{K}\left|y_{i}(k)\right|^{2}$.

In cases, where the PUs are communicating with the PBS, the transmitted signal is also being received by the sensing devices which are located within the transmission range of the PU. Therefore, the sensors periodically sense channel $i$ and obtain the corresponding test statistics, i.e., energy levels, and the hypothesis test is then performed based on the measured parameters and the system defined parameters.

The performance of the spectrum sensing techniques is characterized by false alarm and miss detection probabilities. False alarm is referred to the cases, where $H_{1}$ is decided while the channel is in fact available. Similarly, miss detection is defined as the cases, where $H_{0}$ is decided while the channel is in fact unavailable. For a channel $i$, the probability of false alarm, and miss detection are represented by $P_{f, i}$, and $P_{m, i}$, respectively, and detection probability is defined as $P_{d, i}=1-P_{m, i}$. The lower the detection probability, the higher is the chance of collision between PU and SU transmission; thus lower is the the system spectral efficiency. Similarly, having a higher false alarm results in under-utilization of the practically available primary spectrum by the SUs [2].

The miss detection and false alarm probabilities are obtained as Chi-squared distribution with $2 K$ degrees of freedom, however it is shown, according to the central limit theorem, that for a large number of independent and identically distributed (i.i.d) samples $(K>40)$ obtained from primary transmitter, the cumulative density function (CDF) of the estimated energy can also be approximated by a normal distribution, see, e.g., [27]. In such cases, the false alarm and detection probabilities are [3]:

$$
\begin{aligned}
P_{f, i}\left(\varepsilon_{i}, T_{s, i}\right) & =\operatorname{Pr}\left(E_{i}(y)>\varepsilon_{i} \mid H_{0}\right) \\
& \approx \mathrm{Q}\left(\left(\frac{\varepsilon_{i}}{\sigma_{w}^{2}}-1\right) \sqrt{T_{s, i} f_{s}}\right),
\end{aligned}
$$

and

$$
\begin{aligned}
P_{d, i}\left(\varepsilon_{i}, T_{s, i}\right) & =\operatorname{Pr}\left(E_{i}(y)>\varepsilon_{i} \mid H_{1}\right) \\
& \approx \mathrm{Q}\left(\left(\frac{\varepsilon_{i}}{\sigma_{w}^{2}}-\gamma_{i}-1\right) \sqrt{\frac{T_{s, i} f_{s}}{2 \gamma_{i}+1}}\right),
\end{aligned}
$$

where

$$
\gamma_{i}=\frac{\mathrm{E}\left[\left|x_{i}\right|^{2}\right]\left|g_{i}\right|^{2}}{\sigma_{w}^{2}}
$$

is the average received SNR of the PUs signal on channel $i$. Here, $\varepsilon_{i}$ and $T_{s, i}$ are the energy detection threshold and sensing duration for the sensing devices. Moreover, $\varepsilon_{i}$ and $T_{s, i}$ are the design parameters and they represent the trade-off between $P_{f, i}\left(\varepsilon_{i}, T_{s, i}\right)$, and $P_{m, i}\left(\varepsilon_{i}, T_{s, i}\right)=1-P_{d, i}\left(\varepsilon_{i}, T_{s, i}\right)$ which is often referred to as receiver operating characteristics (ROC) curve [28].

\section{Zone-Based Cooperative Spectrum Sensing}

In the proposed method, spectrum sensors report the locally sensed channel decision to their corresponding ZAs. ZAs then transmit their aggregated decision to the SBS. In cases where the SUs request for the new channel, an available channel from $\{1, \ldots, N\}$ is granted to the SU. Therefore, the efficiency of the proposed method depends on the accurate detection of the PU activity on each channel rather than sensing duration, since in the proposed method, sensors are, in fact, independent from the secondary network.

The logical AND rule is implemented at the ZAs which is applied on the sensing information collected from individual sensors in its corresponding zone. Based on AND rule, for a channel to be available in a zone all sensors located in a zone must unanimously agree on the channel availability. In other words, if any sensor in a given zone observes channel $i$ as busy, channel $i$ is considered busy thus the SUs located in that zone are not granted access to channel $i$ by the SBS. This rather pessimistic strategy is designed to best protect the active PUs within the zone. As a result, the achievable spectral efficiency in this case acts as a lower bound to the maximum achievable spectral efficiency. Other techniques, e.g., k-out-of$\mathrm{N}$, can be applied depending on the interference suppression capability of the primary system. In addition, using this fusion method maintains the mathematical tractability to obtain the sensing thresholds later in the paper. Here, SBS may also act as ZA in cases where the cell size is small such that sensors have direct communication with the SBS.

Corresponding to each channel, one bit information is generated by each sensor, where 0 indicates the channel is available and, 1 otherwise. For instance, if there are 10 sensors in a zone monitoring a total of 128 channel, for each sensing period, a total 1280 bits of signaling is transmitted in that zone. ZA then feeds back the channel availability to the DFC as a binary vector, where each entry shows the availability of 


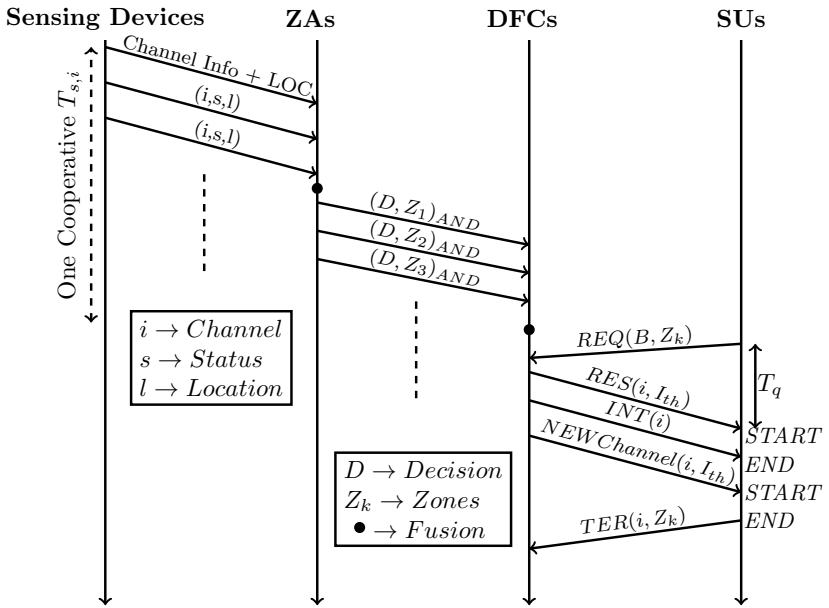

Fig. 2. Signaling diagram of the zone-based cooperative spectrum sensing.

the corresponding channel in that zone. DFC then allocates channels to maximize micro-spectrum-reuse.

Signaling diagram for the proposed zone-based cooperative sensing techniques is shown in Fig. 2. The sensing devices are synchronized and they sense the channels periodically. Therefore, every sensing device is programmed to sense the channels and reports its sensing decision back to its corresponding ZA. The proposed protocol in this paper is based on providing best-effort service to the SUs. The SU which requires access to the channel transmits a request message (REQ) to the SBS including its required bandwidth $(B)$ as well as its corresponding ZIDs $\left(Z_{k}\right)$. The received ZIDs by each SU act as a location pointer.

The DFC then allocates channels, $i \in\{1, \ldots, N\}$, to the SU in that zone (if any) as well as corresponding thresholds, $I_{t h}$. Here $I_{t h}$ is a system defined parameter and it is set by primary system according to their capacity to suppress the inter-zone interference, via a response message (RES). Furthermore, the DFC is able to incorporate other information in its decision making, such as channel and traffic variations. Thus DFC has a potential to act as a knowledge-based/expert entity which keeps record of relevant primary channel information such as traffic activities and load variations, transmission power, and channel power gain.

The SUs then start communicating on the allocated channels while constantly checking the ZIDs. Here, we adopt the coexistence beacon protocol as in [29] in which channel information is embedded in the transmission. In our proposed method and later in the simulation, a unique identity is set for the PUs and SUs which is also embedded in their transmitted signal. As soon as a PU starts transmission, then using this unique identity field, the sensing devises are capable of recognizing that the detected signal is in fact from a PU transmitter. The monitoring network continuously senses the channels. Therefore, if a PU starts transmitting on a given channel, the SUs transmission on that channel is immediately stopped and other available channels, if any, will be allocated to that SU. Similarly, if a SU moves into another zone, i.e., its corresponding ZID is changed, the allocated channel in its original zone is released and a new channel, if available, is

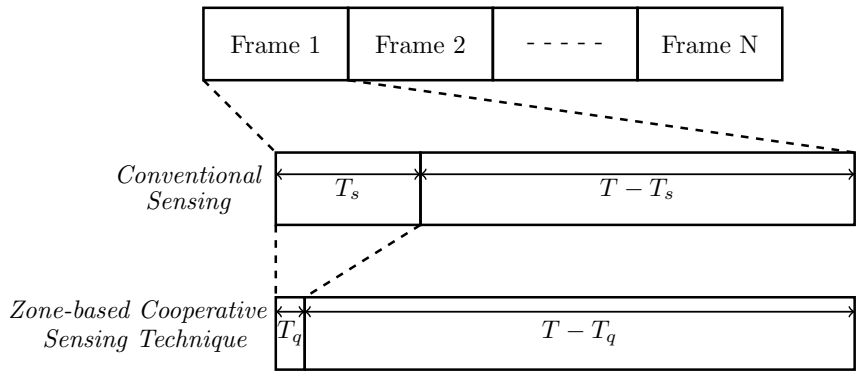

Fig. 3. The time frame in the proposed method consists of the query duration $\left(T_{q}\right)$, and transmission duration $\left(T-T_{q}\right)$. In the conventional sensing, a frames consists of the sensing duration, $T_{s}$, and transmission duration ( $T-$ $\left.T_{s}\right)$

allocated to the SU in its new zone. Alternatively, to identify whether a detected signal is from a PU transmitter, interframe quiet period (IFQP) protocol [29] can also be used. In such cases, the DFC sends an interrupt message (INT) to the SU to immediately release the allocated channel(s). If SU still requires access and previously allocated channels are no longer available, a NEW message is sent by the DFC allocating new channel(s) (if available), where NEW message has same parameters as RES message. In cases, where the SU does not require access anymore, a terminating message (TER) is sent to the DFC to release the corresponding channel $i \in\{1, \ldots, N\}$ within zone $Z_{k}$.

In the proposed protocol for the zone-based cooperative spectrum sensing, the required signaling between the sensors and the ZAs, and similarly ZA and the DFC is designed to be very limited to reduce the spectrum resources allocated to the monitoring network.

Note that a given channel might be available in more than one zones thus based on the proposed method in this paper, micro-spectrum-reuse is expected in multiple zones inside the SBS coverage.

\section{A. Off-Loading and Sensing Latency}

Off-loading of the spectrum sensing activities to the independent sensing devices has a direct implication on the latency, and thus on the system throughput. Due to a separate sensing network which maintains almost real-time primary channel availability status, the corresponding channel allocation latency in the secondary user is significantly reduced comparing to the cases without the spectrum monitoring network. This has been investigated later in Section IV and validated through the simulations in Section V.

The time frames structure of the proposed method and that of the conventional sensing are shown in Fig. 3. Here, $T_{s}$ is the sensing duration for the conventional spectrum sensing and $T_{q}$ is the duration of the required communication between the secondary system and the secondary base station. Hereafter, we refer to $T_{q}$ as the query time, where $T_{q}<<T_{s}$. The low latency of the proposed signaling method is due to substituting the sensing duration $T_{s}$ with $T_{q}$. The extra transmission time, $T_{s}-T_{q}$, results in increasing the total system spectral efficiency and its corresponding cost is deploying the spectrum monitoring network. Therefore, careful analysis is required to 
evaluate whether the gain on the spectral efficiency dominates the costs of deploying the monitoring network.

Without sensing devices, a portion of the frame duration, i.e., $T_{s}$, must be sacrificed for spectrum sensing by the SUs. As a result, a shorter time is available to the SUs for data transmission. Therefore, off-loading the sensing task to the sensing devices significantly increases transmission durations without reducing the sensing accuracy. The optimal sensing duration, $T_{s}$ is not defined in WRAN standard [29], however it is shown in [3] that the optimal $\frac{T_{s}}{T}$ is $4 \%$ to $5 \%$. In the proposed method, $\frac{T_{q}}{T_{s}}$ is chosen to be less than $1 \%$.

Because of the independent spectrum sensing network, the sensing devices are able to sense the channel throughout the frame duration. Therefore, using the zone-based cooperative sensing protocol enables simultaneous sensing, in the monitoring network, and data transmission at the secondary system. In this case, the only time interval required for obtaining the availability of the channel is $T_{q}$ which is the duration of signaling between REQ messages sent by the SU and RES message sent by the DFC. The signaling duration in the proposed method is a very small fraction of sensing duration of the conventional approach of spectrum sensing.

\section{Sensing Design}

\section{A. Spectrum Sensing Accuracy}

Inaccurate sensing either negatively affects the primary system performance through creating interference (in cases of miss detection), or results in a lower spectral efficiency in the secondary network by missing an actual access opportunity (in cases of false alarm). To investigate the sensing accuracy, here we simply assume that the sensors are uniformly distributed in the network coverage area.

Lemma 1. In a monitoring network with $Z$ ZAs/cell indexed by $z=1, \ldots, Z$ and $S$ cooperative $S B S$ indexed by $s=$ $1, \ldots, S$, the probability of accurate sensing for equiprobable hypotheses channels [30], $i \in\{1, \ldots, N\}$, at the SBS is:

$P_{c s, i}^{(S B S)} \triangleq 1-\left[\left\{1-P_{d}\left(\varepsilon_{i}, T_{s, i}\right)\right\}^{Z}+\left\{P_{f}\left(\varepsilon_{i}, T_{s, i}\right)\right\}^{Z}\right]^{S}, \forall i$.

Proof. See Appendix A.

Remark 1. The probabilities for hypotheses $H_{0}$, and $H_{1}$ are denoted by $P_{H_{0}}$, and $P_{H_{1}}$, respectively. Equiprobable channel assumption indicates that half of the channels are busy at any observation window. However, the analytical and simulation results in the next sections in this paper are equally credible for other scenarios, for instance, unutilized, i.e., $P_{H_{0}}<<0.5$, underutilized, i.e., $P_{H_{0}}>0.5$, and crowded, i.e., $P_{H_{0}}>0.9$ channels. This assists obtaining analytical solutions in terms of detection threshold, and normalized throughput.

Lemma 1 indicates that $P_{c s, i}$ depends on probabilities of miss detection and false alarm, as well as the number of ZAs and sensors in each zone. This provides two new degrees of freedom which could be exploited to improve the sensing accuracy. In practical systems, the summation of the two terms inside the bracket in (3) constitutes a small value for a given sensing device. This is due to the fact that miss detection and false alarm probabilities cannot independently adopt arbitrary values as they follow the corresponding sensors' ROC.

Note that in (3), $P_{c s, i} \in[0,1]$ which can be obtained by varying the operating points in ROC curve within the limits, i.e., $P_{m}\left(\varepsilon_{i}, T_{s, i}\right) \leq 0.5$, and $P_{f}\left(\varepsilon_{i}, T_{s, i}\right) \leq 0.5$. These cases are also described in detail in $(5 \mathrm{c}),(5 \mathrm{~d})$ in problem $\mathcal{P}_{1}$. By applying these constraints, it is assured that the probability of correctly sensing the channel stays within the feasible range and therefore value of $P_{c s, i}$ stays within 0 and 1 . This also ensures the protection from system failure due to the bad detectors. Therefore, the worst detection cases, e.g., $P_{f}\left(\varepsilon_{i}, T_{s, i}\right) \geq 0.5$ and $P_{m}\left(\varepsilon_{i}, T_{s, i}\right) \geq 0.5$, are excluded in the proposed method. As a result, if a channel is badly detected, the resources will not be allocated by the SBS to any user to protect the primary users from probable interference.

\section{B. Optimal Sensing for Maximum Spectral Efficiency}

Here, we formulate the system function as an optimization problem with the objective of maximizing the spectral efficiency. In addition, $R_{i}^{00}$, and $R_{i}^{01}$ are the SUs' throughput conditioned over hypotheses $H_{0}$, and $H_{1}$, respectively. Therefore, based on conditional probability of correctly sensing the channel and [3], [4], the achievable throughput is obtained as $\frac{T-T_{s, i}}{T}\left(P_{c s, i \mid H_{0}} P_{H_{0}} R_{i}^{00}+P_{c s, i \mid H_{1}} P_{H_{1}} R_{i}^{01}\right)$. Assuming equiprobable hypotheses [30], the secondary system throughput for channel $i$ is reduced to

$$
R\left(\varepsilon_{i}, T_{s, i}\right)=\frac{T-T_{s, i}}{T}\left(P_{c s, i} P_{H_{0}} R_{i}^{00}+P_{c s, i} P_{H_{1}} R_{i}^{01}\right), \forall i .
$$

Here, $P_{c s, i}$ represents the measure of spectral efficiency of the secondary system. A higher sensing accuracy contributes towards a higher spectral efficiency thus improves system throughput.

For a special case of $Z=S=1$, using (3) and (4) the total secondary system throughput, $R\left(\varepsilon_{i}, T_{s, i}\right)$, is

$$
\frac{T-T_{s, i}}{T}\left(\left(1-p_{f}\right) P_{H_{0}} R_{i}^{00}+\left(1-p_{f}\right) P_{H_{1}} R_{i}^{01}-K_{L}\right),
$$

where, $\left.K_{L}=\left(1-p_{d}\right) P_{H_{0}} R_{i}^{00}+\left(1-p_{d}\right) P_{H_{1}} R_{i}^{01}\right)$ is the throughput loss due to the miss detection $\left(P_{m}>0\right)$. Note that if $P_{m} \rightarrow 0$, then $K_{L} \rightarrow 0$.

For given values of $Z$ and $S$, the optimal sensing parameters are obtained via the following optimization problem.

Problem $\mathcal{P}_{1}$ :

$$
\begin{array}{cl}
\max _{\varepsilon_{i}, T_{s, i}} & R\left(\varepsilon_{i}, T_{s, i}\right), \\
\text { s.t. } & I_{p}\left(\varepsilon_{i}, T_{s, i}\right) \leq \bar{I}_{t h}, \\
& P_{m}\left(\varepsilon_{i}, T_{s, i}\right) \leq \bar{P}_{m}, \\
& P_{f}\left(\varepsilon_{i}, T_{s, i}\right) \leq \bar{P}_{f}, \forall i
\end{array}
$$

where

$$
I_{p}\left(\varepsilon_{i}, T_{s, i}\right)=\sum_{i} P_{m, i}\left(\varepsilon_{i}, T_{s, i}\right) P_{t, s}\left|g_{i}\right|^{2}
$$


is the aggregated interference received at the PUs. For channel $i,(5 b)$ ensures that the received interference remains below the given threshold level, $\bar{I}_{t h}$. This will protect the PUs against the potential sensing errors [31]. In addition, the minimum detection probability of "spectrum holes" is enforced by $(5 \mathrm{c})$ and (5d). In $\mathcal{P}_{1}, P_{t, s}$ is the SU's maximum transmit power, $g_{i}$ is the channel gain between the secondary transmitter and the primary receiver, and $\bar{P}_{m}$, and $\bar{P}_{f}$ are the maximum miss detection, and false alarm probabilities, respectively. These parameters are provided by the related communication standards, see, e.g., [29].

In $\mathcal{P}_{1}, P_{H_{0}} R_{i}^{00}+P_{H_{1}} R_{i}^{01}$ is constant during a time frame duration, $T$. Moreover, in the proposed method, $T_{s, i}=T_{q} \ll$ $T$, therefore $\frac{T-T_{s, i}}{T}$ is almost constant (See Fig. 3) which is referred to as $T_{T x}$ throughout this paper. Consequently, the only optimization parameter in $\mathcal{P}_{1}$ is $P_{c s, i}$, which is a function of $\varepsilon_{i}$, and $T_{s, i}$.

Based on the above, $\mathcal{P}_{1}$ is then reduced to the following optimization problem:

Problem $\mathcal{P}_{2}$ :

$$
\begin{array}{rl}
\max _{\varepsilon_{i}, T_{s, i}} & 1-\left[\left\{1-P_{d}\left(\varepsilon_{i}, T_{s, i}\right)\right\}^{Z}+\left\{P_{f}\left(\varepsilon_{i}, T_{s, i}\right)\right\}^{Z}\right]^{S}, \\
\text { s.t. } & \sum_{i} P_{m}\left(\varepsilon_{i}, T_{s, i}\right) P_{t, s}\left|g_{i}\right|^{2} \leq \bar{I}_{t h}, \\
& P_{m}\left(\varepsilon_{i}, T_{s, i}\right) \leq \bar{P}_{m}, \\
& P_{f}\left(\varepsilon_{i}, T_{s, i}\right) \leq \bar{P}_{f}, \forall i .
\end{array}
$$

To obtain the solutions of $\mathcal{P}_{2}$, using the Lemmas in Appendices B-D, (7d) is approximated by $\sigma_{w}^{2} \leq \varepsilon_{i} \leq\left(1+\gamma_{i}\right) \sigma_{w}^{2}$.

To further simplify $\mathcal{P}_{2}$ we use the following Lemma in [32].

Lemma 2. [32] For $\sigma_{w}^{2} \leq \varepsilon_{i} \leq\left(1+\gamma_{i}\right) \sigma_{w}^{2}, P_{m}\left(\varepsilon_{i}, T_{s, i}\right)$ and $P_{f}\left(\varepsilon_{i}, T_{s, i}\right)$ are decreasing convex functions of $T_{s, i}$.

It is also shown in [32] that, given the stated conditions of Lemma in Appendix B, $\frac{\partial^{2} P_{m}\left(\varepsilon_{i}, T_{s, i}\right)}{\partial^{2} T_{s, i}}<0$, and $\frac{\partial^{2} P_{f}\left(\varepsilon_{i}, T_{s, i}\right)}{\partial^{2} T_{s, i}}>$ 0 . It is further straightforward to prove the following Lemma based on Lemmas 4, 5, and 6 in Appendices B-D.

Lemma 3. For a given $T_{s, i}$, if $P_{m}\left(\varepsilon_{i}, T_{s, i}\right) \leq 0.5$, and $P_{f}\left(\varepsilon_{i}, T_{s, i}\right) \leq 0.5$, then $P_{m}\left(\varepsilon_{i}, T_{s, i}\right)$, and $P_{f}\left(\varepsilon_{i}, T_{s, i}\right)$ are both convex functions of $\varepsilon_{i}$.

Based on Lemmas 2, 3, and those in Appendices B-D, we then conclude that both $P_{m}\left(\varepsilon_{i}, \bar{T}_{s, i}\right)$ and $P_{f}\left(\varepsilon_{i}, \bar{T}_{s, i}\right)$ are convex functions of $\varepsilon_{i}$, where sensing duration is fixed at $\bar{T}_{s, i}$ under the conditions to protect the PUs.

Here, the conditions to maximize the throughput are: $P_{d}\left(\varepsilon_{i}, T_{s, i}\right) \geq 0.5$, and $P_{m}\left(\varepsilon_{i}, T_{s, i}\right) \leq 0.5$, which are the requirements of IEEE 802.22 standards [29].

Based on the above, $\mathcal{P}_{2}$ is approximated as the following.

\section{Problem $\mathcal{P}_{3}$ :}

$$
\begin{array}{ll}
\max _{\varepsilon_{i}} & 1-\left[\left\{1-P_{d}\left(\varepsilon_{i}\right)\right\}^{Z}+\left\{P_{f}\left(\varepsilon_{i}\right)\right\}^{Z}\right]^{S}, \\
\text { s.t. } & \sum_{i} P_{m}\left(\varepsilon_{i}, T_{s, i}\right) P_{t, s}\left|g_{i}\right|^{2} \leq \bar{I}_{t h}, \\
& \sigma_{w}^{2} \leq \varepsilon_{i} \leq\left(1+\gamma_{i}\right) \sigma_{w}^{2}, \forall i .
\end{array}
$$

In $\mathcal{P}_{3},(8 \mathrm{c})$ is convex under the stated conditions in Lemma 4. The interference constraint at the PU, (8b), is due to the imperfect channel sensing, where $\left|g_{i}\right|^{2}$ is the gain of channel $i$. Here, $P_{t, s}>0$ is the transmission power of the SU and $P_{m, i}\left(\varepsilon_{i}, \bar{T}_{s, i}\right)$ is a convex function of $\varepsilon_{i}$ under the condition given in Lemma 4. Since non-negative sum of convex functions is a convex function in the same domain, the interference constraint is also a convex function of $\varepsilon_{i}$. To show the convexity of $\mathcal{P}_{3}$, we further need to investigate (8a). Note that throughout this paper $P_{m(f)}\left(\varepsilon_{i}, \bar{T}_{s, i}\right)$ and $P_{m(f)}\left(\varepsilon_{i}\right)$ are interchangeably used for brevity.

Corollary 1. In the zone-based cooperative spectrum sensing, for any combination of $S$ and $Z$, the throughput, $(8 a)$, is a concave function of $\varepsilon_{i}$.

\section{Proof. See Appendix E.}

Based on the above, $\mathcal{P}_{3}$ is a convex optimization problem.

\section{Optimal Detection Threshold}

Here, we utilize Lagrangian method to find the solutions of $\mathcal{P}_{3}$. We then apply Lagrange duality property as in [33]. The Lagrangian function corresponding to $\mathcal{P}_{3}$ is

$$
\begin{gathered}
\mathcal{L}\left(\varepsilon_{i}, \lambda_{1}, \boldsymbol{\lambda}_{\mathbf{2}}, \boldsymbol{\lambda}_{\mathbf{3}}\right)=1-\left[\left\{1-P_{d}\left(\varepsilon_{i}\right)\right\}^{Z}+\left\{P_{f}\left(\varepsilon_{i}\right)\right\}^{Z}\right]^{S} \\
+\lambda_{1}\left(I_{t h}-\sum_{i=1}^{N} P_{m} P_{t, s}\left|g_{i}\right|^{2}\right)+\sum_{i=1}^{N} \lambda_{2 i}\left(\varepsilon_{\max }-\varepsilon_{i}\right) \\
+\sum_{i=1}^{N} \lambda_{3 i}\left(\varepsilon_{i}-\varepsilon_{\text {min }}\right),
\end{gathered}
$$

where, $\varepsilon_{\max }=\left(1+\gamma_{i}\right) \sigma_{w}^{2}, \varepsilon_{\min }=\sigma_{w}^{2}$, and $\lambda_{1}, \boldsymbol{\lambda}_{\mathbf{2}}, \boldsymbol{\lambda}_{\mathbf{3}}$ are non-negative Lagrangian dual variables corresponding to the constraints. Here, $\lambda_{1}$ is scalar because channel $i$ accessed exclusively by only one PU. The interference constraint protects the PUs on channel $i=1, \ldots, N$ in case of miss detection. Similarly, $\boldsymbol{\lambda}_{\mathbf{2}}$ and $\boldsymbol{\lambda}_{\mathbf{3}}$ are the Lagrangian multipliers associated with detection threshold constraints. Throughput this paper, vectors are presented using bold fonts.

The corresponding duality gap is expected to be very close to zero as $\mathcal{P}_{3}$ is concave and the Slater's condition [33] is satisfied. The KKT conditions for any set of $\varepsilon_{i}^{*}, \lambda_{1}, \boldsymbol{\lambda}_{\mathbf{2}}, \boldsymbol{\lambda}_{\mathbf{3}}$ are [33]:

$$
\begin{aligned}
& \nabla \mathcal{L}\left(\varepsilon_{i}^{*}, \lambda_{1}^{*}, \boldsymbol{\lambda}_{\mathbf{2}}^{*}, \boldsymbol{\lambda}_{\mathbf{3}}^{*}\right)=\mathbf{0}, \\
& I\left(\varepsilon_{i}^{*}\right) \leq I_{t h}, \\
& \lambda_{1}^{*}>0, \boldsymbol{\lambda}_{\mathbf{2}}^{*} \succ \mathbf{0}, \boldsymbol{\lambda}_{\mathbf{3}}^{*} \succ \mathbf{0}, \\
& \lambda_{1}^{*}\left(I_{t h}-\sum_{i=1}^{N} P_{m, i} P_{t x}\left|g_{i}\right|^{2}\right)=0, \\
& \sum_{i=1}^{N} \lambda_{2 i}\left(\varepsilon_{\max }-\varepsilon_{i}^{*}\right)=0, \\
& \sum_{i=1}^{N} \lambda_{3 i}\left(\varepsilon_{i}^{*}-\varepsilon_{\min }\right)=0, \quad \forall i .
\end{aligned}
$$


Here, we follow a similar approach as in [31] to obtain the solutions. If the condition $\sigma_{w}^{2}<\varepsilon_{i}<\left(1+\gamma_{i}\right) \sigma_{w}^{2}$ holds, the constraint (10b) becomes linear, i.e., $I\left(\varepsilon_{i}^{*}\right)=I_{t h}$. Therefore, for any $\lambda_{1}^{*} \geq 0, \lambda_{1}^{*}\left(I_{t h}-I\left(\varepsilon_{i}^{*}\right)\right)=0$.

In the considered multi-channel scenario, we now assume that the channels are identically distributed and sensed similarly, thus the results obtained are valid for all channels, $i \in\{1, \ldots, N\}$. Therefore, we hereafter drop the channel index, $i$, for brevity. From the Lagrangian stationary point, (10a), we get

$$
\frac{\partial \mathcal{L}\left(\varepsilon^{*}, \lambda_{1}^{*}, \boldsymbol{\lambda}_{2}^{*}, \boldsymbol{\lambda}_{\mathbf{3}}^{*}\right)}{\partial \varepsilon}=0 .
$$

If both $Z$ and $S$ vary, then it is not easy to obtain a closed form solution for $\mathcal{P}_{3}$. Instead, we solve this problem separately for different numbers of $Z$ and $S$ similar to the approach used in proving Corollary 1. Here, $\varepsilon_{S}$ is obtained which is defined as sensing detection threshold for all channels, where $S$ is constant. Similarly, $\varepsilon_{Z}$ is then obtained which is defined as sensing detection threshold for all channels, where $Z$ is constant. We then show that the optimal detection threshold is a linear combination of $\varepsilon_{S}$ and $\varepsilon_{Z}$.

The optimal detection threshold for various design scenario has been summarized in Table I. In the following, we investigate each scenario in detail.

1) Scenario $1(Z=1, S=1)$ : In this case, (11) is rewritten as

$$
\begin{aligned}
\frac{\partial \mathcal{L}_{1}\left(\varepsilon^{*}, \lambda_{1}^{*}\right)}{\partial \varepsilon}= & \frac{\partial}{\partial \varepsilon}\left(T_{T x}\left[1-\left(P_{m}(\varepsilon)+P_{f}(\varepsilon)\right)\right]\right. \\
& \left.+\lambda_{1}\left(I_{t h}-P_{m}(\varepsilon) P_{t, s}|g|^{2}\right)\right)=0,
\end{aligned}
$$

which results in the following equation:

$$
T_{T x} \frac{\partial P_{d}(\varepsilon)}{\partial \varepsilon}+\lambda_{1} P_{t, s} \frac{\partial P_{d}(\varepsilon)}{\partial \varepsilon}=T_{T x} \frac{\partial P_{f}(\varepsilon)}{\partial \varepsilon} .
$$

To find the solution, we utilize $\frac{\partial P_{d}(\varepsilon)}{\partial \varepsilon}$ and $\frac{\partial P_{f}(\varepsilon)}{\partial \varepsilon}$ obtained in Lemma 5, and Lemma 6, respectively. For a given $T_{s}$, straightforward mathematical derivations result in a closed form expression for the optimal SNR threshold for all channels

$$
\varepsilon_{S(Z)}^{*}=\frac{\sigma_{w}^{2}}{2 \gamma}\left[\gamma_{c}+\frac{2}{f_{s} T_{s}} \ln \left(\frac{T_{T x}}{T_{T x}+\lambda_{1} P_{t, s}|g|^{2}}\right)\right],
$$

where $\gamma_{c}=(\gamma+1)^{2}-1$.

2) Scenario $2(Z=2, S=1)$ : In this case, similar to (12) amd (13) and straight mathematical derivation, we get the optimum SNR threshold for any channel as

$$
\varepsilon_{S}^{*}=\frac{\sigma_{w}^{2}}{2 \gamma}\left[\gamma_{c}+\frac{2}{f_{s} T_{s}} \ln \left(\frac{2 \bar{P}_{f} T_{T x}}{2 \bar{P}_{m} T_{T x}+\lambda_{1} P_{t, s}|g|^{2}}\right)\right] .
$$

Here, $\varepsilon_{S}^{*}$ is the optimum SNR threshold valid for the frame duration $T$.

3) Scenario $3(Z=3, S=1)$ : Similar to the above, here we get

$$
\varepsilon_{S}^{*}=\frac{\sigma_{w}^{2}}{2 \gamma}\left[\gamma_{c}+\frac{2}{f_{s} T_{s}} \ln \left(\frac{3 \bar{P}_{f}^{2} T_{T x}}{3 \bar{P}_{m}^{2} T_{T x}+\lambda_{1} P_{t, s}|g|^{2}}\right)\right] .
$$

Finally based on the results above, and following the same line of argument as in Corollary 1, for a fixed $S$ and any number of ZAs, i.e., $z=1, \ldots, Z$, we can generalize the optimal SNR threshold as shown in (17).

4) Scenario $4(Z=1, S=2)$ : In this case, at a particular time and location, a SBS may receive sensing information from more than one ZAs. In this scenario, similar to the case where $Z$ is variable, we use Lagrangian stationary point, (10a). For $S=2$, it is simple to show that

$$
\begin{aligned}
\varepsilon_{Z}^{*}= & \frac{\sigma_{w}^{2}}{2 \gamma_{i}}\left[(\gamma+1)^{2}-1+\frac{2}{f_{s} T_{s}}\right. \\
& \left.\times \ln \left(\frac{2\left(\bar{P}_{m}+\bar{P}_{f}\right) T_{T x}}{2\left(\bar{P}_{m}+\bar{P}_{f}\right) T_{T x}+\lambda_{1} P_{t, s}|g|^{2}}\right)\right] .
\end{aligned}
$$

5) Scenario $5(Z=1, S=3)$ : Similar to the previous cases, the optimal threshold can be obtained for different values of $S$, for instance $S=3$. Finally, following the same steps as in obtaining (17), we can generalize the optimal solution for any number of SBSs as shown in (18).

Note that in (14)-(19), the miss detection and false alarm maximum tolerable values are selected such that $\bar{P}_{m}<0.5$, and $\bar{P}_{f}<0.5$. The details are discussed in Section IV.

\section{Unified Detection Threshold}

As it is seen above, the optimal values of detection thresholds, $\varepsilon_{S}^{*}$ and $\varepsilon_{Z}^{*}$, both depend on $Z$ and $S$. In addition, due to the random nature of wireless channel the exact number of sensing devices that their sensing information received at ZA cannot be considered fixed. For instance, some sensing devices may fail to communicate with the ZAs and apparently with SBS. In some cases, the communication channel between sensing devices may also undergo deep fading in which the sensing network scenario is changed. Therefore, a unified detection mode is necessary so that the proposed technique works for any possible scenario and various combinations of $Z$ and $S$. Here we propose a linear combination of $\varepsilon_{S}^{*}$ and $\varepsilon_{Z}^{*}$ as follows:

$$
\varepsilon^{*}=\alpha \varepsilon_{S}^{*}+(1-\alpha) \varepsilon_{Z}^{*},
$$

where $\alpha$ is related to $Z$ and $S$ : if $Z<S$ then $\alpha$ is $0<\alpha<$ 0.5 to emphasize on the contribution of $\varepsilon_{Z}^{*}$ comparing to $\varepsilon_{S}^{*}$ in (20). This is simply because $\varepsilon_{Z}^{*}$ is the detection threshold for cases, where $Z<S$. In contrast, where $Z>S$, system sets $0.5<\alpha<1$, so $\varepsilon_{S}^{*}$ contributes more than $\varepsilon_{Z}^{*}$ in $\varepsilon^{*}$. However, in cases where $Z$ and $S$ are equal, system sets $\alpha=$ 0.5 and apparently $\varepsilon_{S}^{*}$ and $\varepsilon_{Z}^{*}$ contribute equally in (20). In the simulations presented in this paper, $\alpha$ is selected within the ranges mentioned above based on the densities of $Z$ and $S$, for instance, when $Z \gg S, \alpha$ is selected on the lower range of $0.5<\alpha<1$. For the cases where $S=0$, and $Z=0$, the system sets $\alpha=1$, and $\alpha=0$, respectively.

In cases where due to the random time varying nature of wireless communication, such as channel fading, interference, hidden terminal problem, etc., either or both of $Z$ and $S$ are equal to zero, then the optimal detection threshold is undefined because $\varepsilon_{S}^{*}$ and $\varepsilon_{Z}^{*}$ are $-\infty$. As a matter of fact, this situation does not normally occur in the proposed model of zone-based 
TABLE I

THE OPTIMAL SNR THRESHOLD FOR DIFFERENT SCENARIOS

\begin{tabular}{|c|c|}
\hline Scenario $1(Z=1, S=1)$ & $\varepsilon_{X}=\frac{\sigma_{w}^{2}}{2 \gamma}\left[\gamma_{c}+\frac{2}{f_{s} T_{s}} \ln \left(\frac{T_{T x}}{T_{T x}+\lambda_{1} P_{t, s}|g|^{2}}\right)\right]$ \\
\hline Scenario 2 $(Z=2, S=1)$ & $\varepsilon_{X}=\frac{\sigma_{w}^{2}}{2 \gamma}\left[\gamma_{c}+\frac{2}{f_{s} T_{s}} \ln \left(\frac{2 \bar{P}_{f} T_{T x}}{2 \bar{P}_{m} T_{T x}+\lambda_{1} P_{t, s}|g|^{2}}\right)\right]$ \\
\hline Scenario 3 $(Z=3, S=1)$ & $\varepsilon_{X}=\frac{\sigma_{w}^{2}}{2 \gamma}\left[\gamma_{c}+\frac{2}{f_{s} T_{s}} \ln \left(\frac{3 \bar{P}_{f}^{2} T_{T x}}{3 \bar{P}_{m}^{2} T_{T x}+\lambda_{1} P_{t, s}|g|^{2}}\right)\right]$ \\
\hline Scenario 4 $(Z=1, S=2)$ & $\varepsilon_{X}=\frac{\sigma_{w}^{2}}{2 \gamma}\left[(\gamma+1)^{2}-1+\frac{2}{f_{s} T_{s}} \times \ln \left(\frac{2\left(\bar{P}_{m}+\bar{P}_{f}\right) T_{T x}}{2\left(\bar{P}_{m}+\bar{P}_{f}\right) T_{T x}+\lambda_{1} P_{t, s}|g|^{2}}\right)\right]$ \\
\hline Scenario 5 $(Z=1, S=3)$ & $\varepsilon_{X}=\frac{\sigma_{w}^{2}}{2 \gamma}\left[(\gamma+1)^{2}-1+\frac{2}{f_{s} T_{s}} \times \ln \left(\frac{3\left(\bar{P}_{m}+\bar{P}_{f}\right)^{2} T_{T x}}{3\left(\bar{P}_{m}+\bar{P}_{f}\right)^{2} T_{T x}+\lambda_{1} P_{t, s}|g|^{2}}\right)\right]$ \\
\hline
\end{tabular}

$$
\varepsilon_{Z}^{*}=\frac{\sigma_{w}^{2}}{2 \gamma}\left[(\gamma+1)^{2}-1+\frac{2}{f_{s} T_{s}} \ln \left(\frac{S\left(\bar{P}_{m}+\bar{P}_{f}\right)^{S-1} T_{T x}}{S\left(\bar{P}_{m}+\bar{P}_{f}\right)^{S-1} T_{T x}+\lambda_{1} P_{t, s}|g|^{2}}\right)\right]
$$

cooperative spectrum sensing but should be considered as a special case to avoid singularities. Here we propose a specific treatment to tackle this issue as described in the following.

According to (17) and (18), $S=0$, and $Z=0$ indicate $\varepsilon_{Z}^{*} \rightarrow-\infty$, and $\varepsilon_{S}^{*} \rightarrow-\infty$, respectively. At the same time, the sensing system controls $\alpha$ to avoid such a condition. Therefore, for $S \rightarrow 0$, sensing system sets $\alpha \approx 1$. Therefore,

$$
\lim _{S \rightarrow 0} \varepsilon_{Z}^{*}(S) \cdot(1-\alpha) \approx 0,
$$

which indicates that optimal detection threshold solely depends on the $\varepsilon_{S}^{*}$ in (20). Similarly, if $Z \rightarrow 0$, the sensing system selects $\alpha \approx 0$, thus,

$$
\lim _{Z \rightarrow 0} \varepsilon_{S}^{*}(Z) \cdot(\alpha) \approx 0
$$

i.e., the optimal detection threshold solely depends on the $\varepsilon_{S}^{*}$ in (20). Using this method, we are able to obtain a unified version of optimal spectrum sensing threshold.

In the next section we provide a step by step algorithm for obtaining an estimation for $\varepsilon^{*}$ based on the above analysis. In obtaining the optimal detection threshold which maximizes the system throughput, we adopt the bisection method.

\section{E. An Algorithm for Estimating $\varepsilon^{*}$}

The proposed method to estimate $\varepsilon^{*}$ is presented in Algorithm 1, where $\gamma, P_{d}(\varepsilon)$, and $P_{f}(\varepsilon)$ are sub-channel dependent parameters which are different for each sub-channel. Here, the channel independent parameters are adjusted to obtain the optimal channel detection threshold such that the system throughput is maximized while the constraints are also satisfied by the spectrum sharing system.

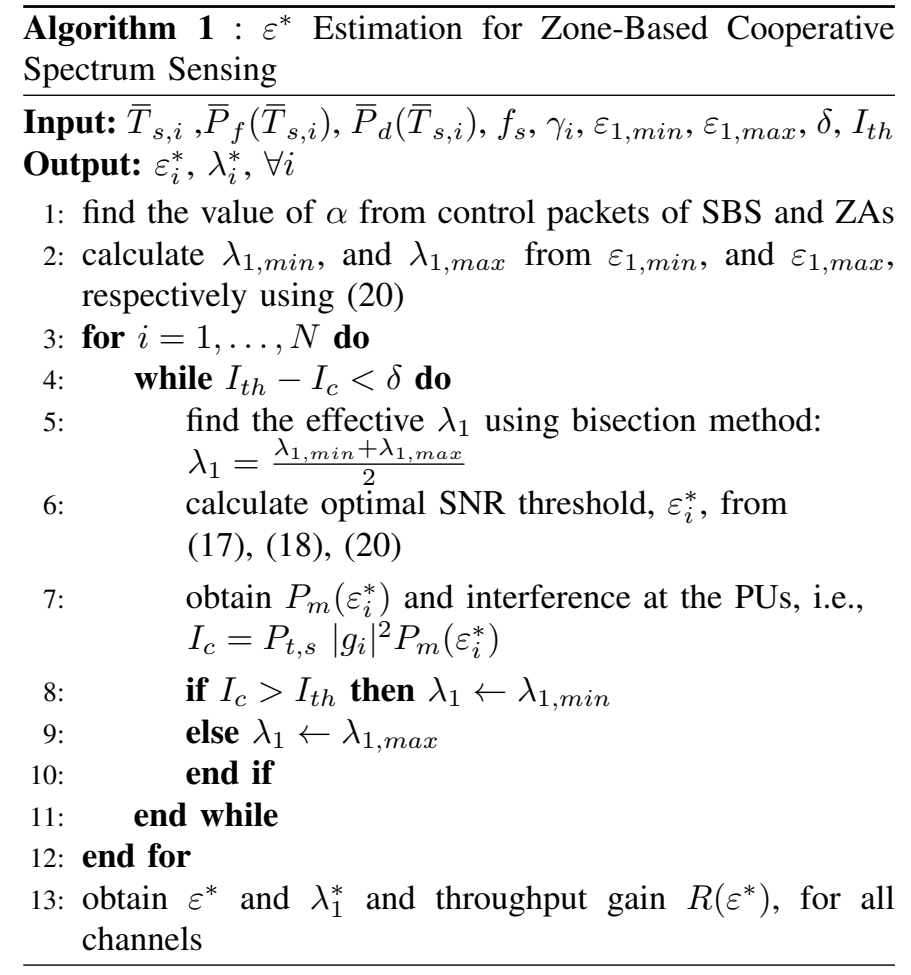

In the proposed method, $T_{s} \ll T$ is a given system parameter thus the optimization variable for each channel is the detection threshold, $\varepsilon_{i}$. We also note that (20) is a monotonically decreasing function of $\lambda_{1}$, i.e., for every $\lambda_{1}^{a}<\lambda_{1}^{b}$, we get $\varepsilon\left(\lambda_{1}^{a}\right)>\varepsilon\left(\lambda_{1}^{b}\right)$. Therefore, bisection method is adopted to find the detection threshold by solving the $\mathcal{P}_{3}$ subject to the constraints in $(8 b)$ and $(8 c)$. 


\section{Simulation Results And Analysis}

In this section, we evaluate the performance of the proposed zone-based cooperative spectrum sensing. We further compare its performance against the benchmark systems including the case where there is no cooperation among the clusters/SBS, and the case where the decisions are diffused using OR/AND rule. We then compare the corresponding resource allocation framework in terms of latency, detection probability, communication activity of primary systems etc. We consider a cognitive radio system, where $N=16$ and $T=100 \mathrm{~ms}$. The mean signaling duration for each $\mathrm{SU}, \mathrm{E}\left[T_{s}\right]$, is maintained at $3 \mathrm{~ms}$. The sampling rate is $f_{s}=20 \mathrm{kSample} /$ Second and therefore the sampling overhead is $f_{s} T_{s}=60$. We also set $\sigma_{w}^{2}=1$. We further assume that $P_{H_{0}}=0.5$. The traffic on the sub-channels is randomly generated and SUs always have data packets ready to be transmitted unless otherwise stated. The channel between primary and secondary system is modeled as Rayleigh fading with scale parameter of 1 . The primary channel protection and spectrum utilization level are defined according to the IEEE 802.22 standard [29] as $\bar{P}_{d} \geq 0.9$, and $\bar{P}_{f} \leq 0.1$, respectively.

\section{A. Comparative Study of Sensing Accuracy}

The proposed zone-based cooperative spectrum sensing, as defined in (3), is validated by considering the appropriate value of detection probability which fulfills the requirement of constraint (5c) as well as WRAN 802.22 standard. Correspondingly, the false alarm probability is obtained from the corresponding ROC curve. Therefore, the instantaneous $P_{d}$ and $P_{f}$ are chosen for a given combination of $S$ and $Z$ to test the spectrum sensing accuracy of the proposed method.

For comparison, we consider $Z=1$ as the conventional cooperative spectrum sensing based on Lemma 1 at the SBSs and therefore there is no channel reusability. The case of higher $Z$ represents the special scenario that the multiple antennas are transmitting at the BSs and the cell is divided into sectors. In this case, each sector can be considered as a single antenna cell and therefore number of ZAs and SBSs are increased. We consider the case $Z=1$ as a benchmarking scenario. In Fig. 4, the normalized system throughput, which is directly related to the system spectral efficiency, is plotted versus the number of zone aggregators for different number of SBSs. Here, it is seen for the case $Z=1$ that the normalized throughput is 0.6 whereas in a zone-based cooperative spectrum sensing method as indicated by $Z \geq 2$, it is significantly improved from 0.9 to 0.96 when ZAs are set to 2 and 3, respectively. This is due to the fact that the proposed method has better channel sensing accuracy than any conventional cooperative channel sensing.

The higher sensing accuracy ensures that no access to that particular channel is granted by the SBS to protect the PUs. The result also provides insight on the rate of spectral efficiency increased by increasing $S$ as a result of the proposed micro-spectrum-reuse technique. Fig. 4 confirms the increase of normalized throughout from 0.9 to 0.98 when cooperative SBSs are increased from 2 to 3 .

Fig. 5 compares the channel sensing accuracy of the proposed method against the non-cooperative as well as channel

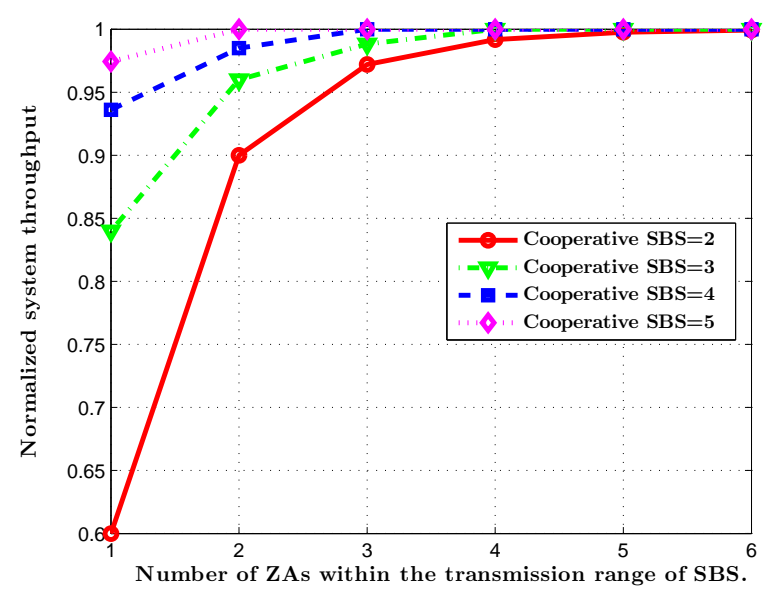

Fig. 4. Normalized throughput vs. different values of $Z$ and $S$.

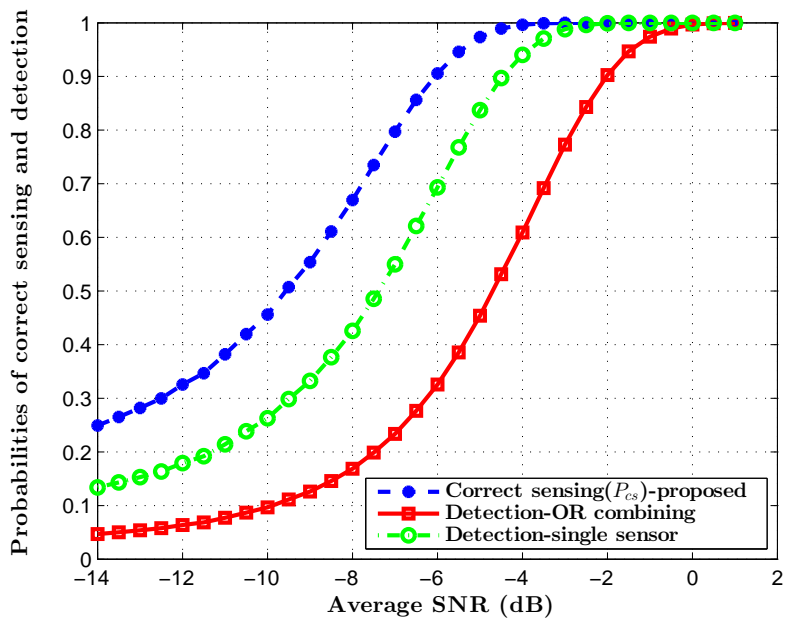

Fig. 5. Probability of correctly detecting the channel $v s$. average received SNR when false alarm rate is fixed.

assignment with cooperative sensing [34] in which the OR fusion methods is implemented for various received SNR. The performance gain in terms of the sensing accuracy is achieved with the expense of installing new sensing infrastructure. In addition to sensing accuracy, it also increases the transmission duration for the SUs which contributes to achieve higher throughput with less system complexity.

In this particular case, the simulation is performed for AWGN channel using QPSK modulation with sampling overhead $T_{s} f_{s}=100$ and $P_{f}$ is no more than 0.1 . The sensing network is set by $Z=3$ and $S=2$ for the proposed method and 3 cooperative sensors for OR fusion method. It can be observed that the correctly sensing probability of the proposed method is improved (0.99) in comparison to non-cooperation (0.6) and when hard decisions are fused with OR method $(0.85)$ at $-4 \mathrm{~dB}$ received SNR. 


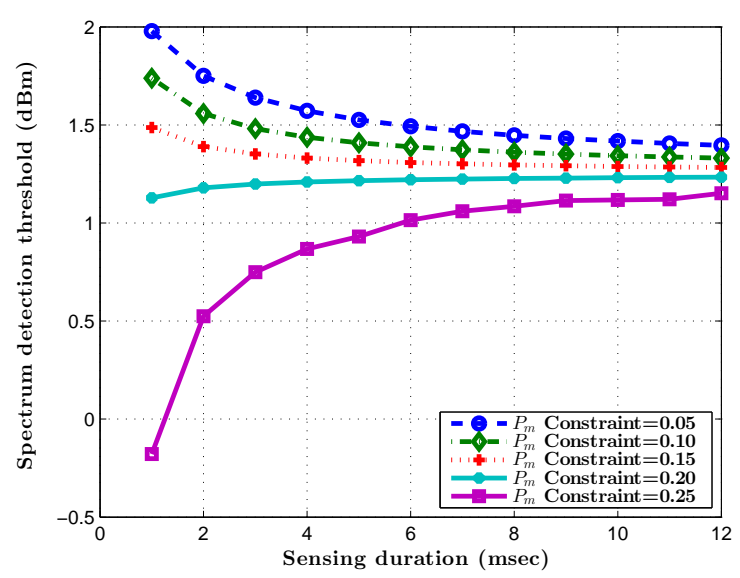

Fig. 6. Optimal spectrum detection threshold vs. sensing duration (latency) for various miss detection constraints.

\section{B. Tradeoff Between Sensing Latency and Detection Threshold}

In Fig. 6, the optimal spectrum detection threshold, $\varepsilon^{*}$, is plotted versus sensing duration at the spectrum sensors for different values of maximum acceptable miss detection probability, where one ZA aggregates channel information from four channel sensors.

As it is seen, for long sensing duration in the secondary system, obtaining the optimal detection threshold deems irrelevant and not related to the maximum acceptable miss detection probability. However in the proposed method, the sensing duration is represented by the short signaling duration, i.e., less than $2 \mathrm{~ms}$ in Fig. 6, the optimal detection threshold must be obtained to improve the system throughput. Therefore, the length of transmission duration does not need to be compromised whilst latency is reduced.

The obvious tradeoff is relaxing the sensing duration $\left(T_{s}>\right.$ $2 \mathrm{~ms}$ ) in which the transmission duration is shorter, and higher latency is then being associated with the spectrum sensing. In contrast, the sensing duration, thus the latency, can be reduced $\left(T_{s}<2 \mathrm{~ms}\right)$, where a higher complexity is expected as the appropriate sensing threshold must be evaluated through the proposed algorithm. Note that in the proposed method the latency associated with the sensing is very small and the cost is limited to the corresponding computational complexity required for evaluating the optimal detection threshold.

\section{Performance Evaluation with Optimal Detection}

Here, we examine the performance of the proposed cooperative spectrum sensing method to maximize the system throughput in which the aggregate interference to the PU is considered to be less than the threshold. In the simulation settings, the conditions in Lemma 2 and Lemmas in Appendices B-D are held. This means that for the simulated system, $\mathcal{P}_{3}$ is convex thus the optimal solutions are (17) and (18).

In the proposed method, the sensing duration, $T_{s}$, is significantly smaller compared to the frame duration, $T$, therefore it is independent of the optimization procedure. However, in

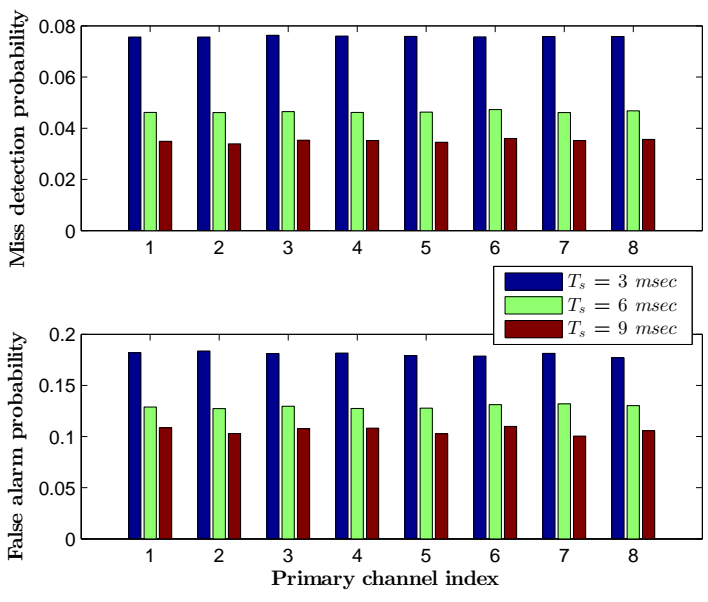

Fig. 7. Probability of miss detection and false alarm of the first eight channels for different values of $T_{s}$.

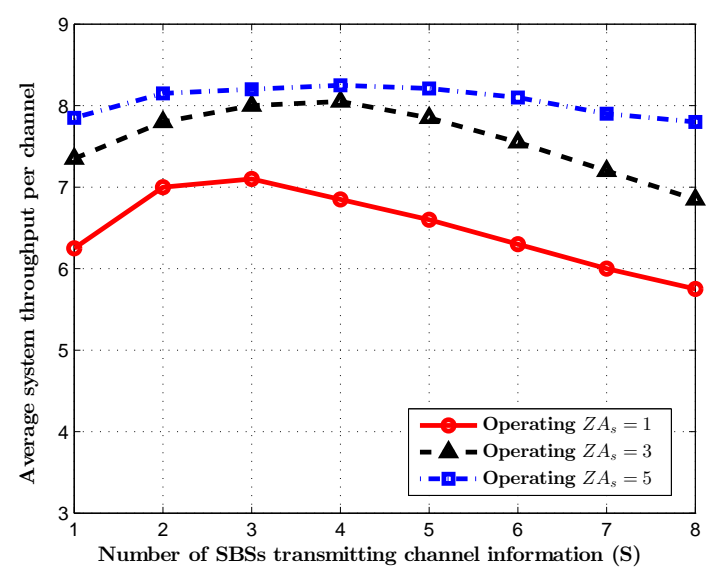

Fig. 8. The average throughput per channel vs. number of SBS.

the conventional spectrum sharing methods, where SUs sense and utilize the ideal channels, optimal choice of $T_{s}$ is crucial. Under the scenario mentioned above, $P_{m}\left(\varepsilon^{*}\right)$, and $P_{f}\left(\varepsilon^{*}\right)$ for an optimal value of detection threshold have been obtained as shown in Fig. 7. While obtaining $P_{m}\left(\varepsilon^{*}\right)$ for an optimal detection threshold, $P_{f}\left(\varepsilon_{i}^{*}\right)$ is kept fixed and vice versa. As expected, the longer the signaling duration, the lower will be the miss detection and false alarm probabilities. In addition, lowering $T_{s, i}$ from $9 \mathrm{~ms}$ to $6 \mathrm{~ms}$ significantly reduces $P_{m}\left(\varepsilon^{*}\right)$ and $P_{f}\left(\varepsilon^{*}\right)$ for all channels. On the other hand, reduction of $T_{s}$ from $6 \mathrm{~ms}$ to $3 \mathrm{~ms}$ does not reduce sensing accuracy in the same proportion. This suggests a way to adjust the signaling duration based on the required spectrum detection accuracy.

In Fig. 8, the average throughput per channel is plotted versus the number of SBSs which transmit the cooperative control packet for the channel detection. We observe that as the number of SBSs are optimal for a given cluster heads, the throughput is maximized. However, lower number of SBS will receive less information about channel availability and, as a result, the throughput per channel decreases. In addition, when 


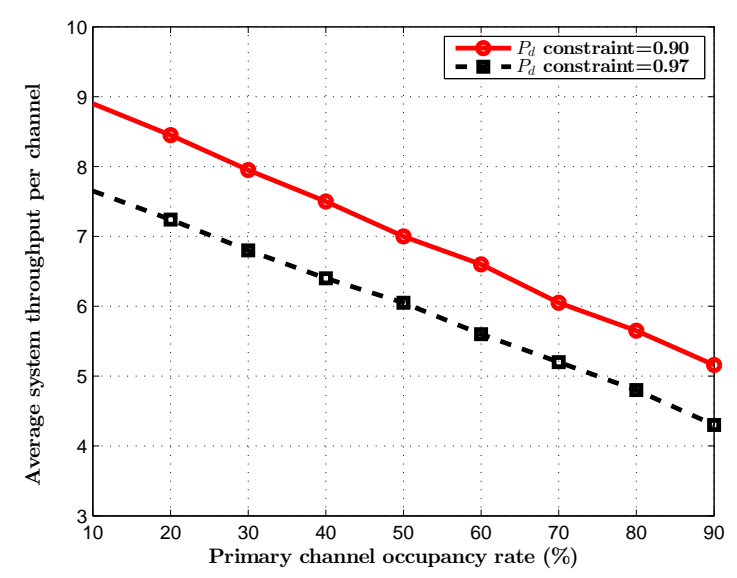

Fig. 9. Average system throughput per channel vs. the primary channel activity for various detection probability constraints.

the number of signaling bits at the SBS increases, secondary system has to perform logical AND operation among large decision variables which results in detecting less opportunities to access the primary channel.

Furthermore the higher the number of ZAs, the higher is the accuracy of channel sensing. Therefore, the system throughput is increased accordingly. For a given simulation setup, the increment of ZAs from 1 to 3 significantly increases per channel throughput in comparison to increment from 3 to 5 .

Fig. 9 shows average throughput per channel versus the primary channel occupancy rate. When the primary channels that can not be accessed by the secondary systems is typically at $10 \%$, the access constraint is relaxed and more channels are available to be shared among SUs. As a result, the system throughput is increased. In contrast, when $90 \%$ of the channels are occupied, SUs have a tight constraint on channel selection and protection of primary systems. In addition, when the probability of detection constraint is relaxed from 0.97 to 0.9 , there is a higher chance to access the channel, in both cases, the channel occupancy rate is linearly decreased with the increasing primary channel activities.

Throughput per channel versus the probability of detection constraint, i.e., $\bar{P}_{d}$, is given in Fig. 10 for various false alarm probabilities. For $\bar{P}_{d}$ lower $(\approx 0.8)$, there is a higher chance of miss detection and therefore the throughput is relatively lower than the case where $\bar{P}_{d}$ is optimal $(\approx 0.9)$. However, by increasing $\bar{P}_{d}$ from 0.9 to 0.99 , the throughput falls down quickly because the detection constraint becomes tight and smaller number of primary channels are available. It has also been observed that as long as $\bar{P}_{f}$ remains within the maximum limit defined in the simulation setup for a given optimal $\bar{P}_{d}$, the throughput is not significantly degraded even if the false alarm constraint is relaxed from $\bar{P}_{f}=0.1$ to 0.2 . Therefore, the optimal choices of $\bar{P}_{d}$ and $\bar{P}_{f}$ are always important for the accurate sensing of the channels and efficiently utilizing them.

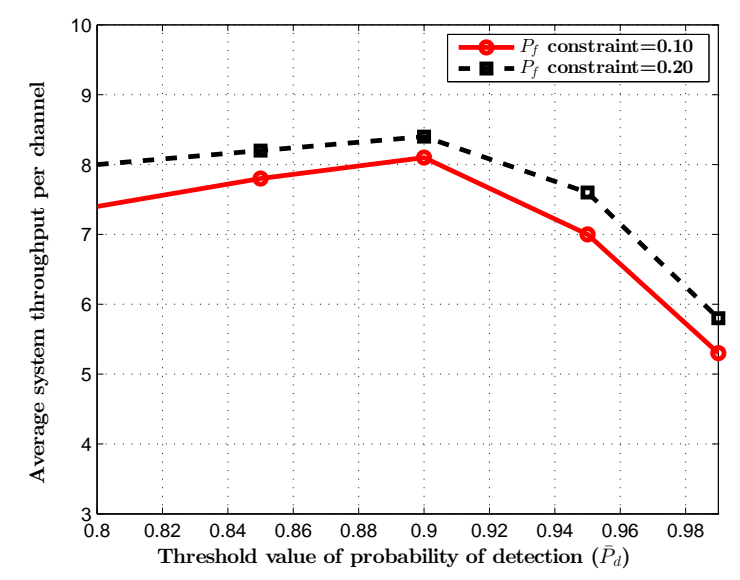

Fig. 10. Average system throughput/channel vs. the probability of detection for various false-alarm probability constraints.

\section{CONCLUSION}

In this paper, we presented a new method for multichannel spectrum sensing through a monitoring network in which the location of the sensor is partly incorporated into channel allocation. We showed that the proposed zone-based cooperative spectrum sensing method increases the sensing accuracy and facilitates higher spectrum reusability thus higher spectrum efficiency. We also developed the techniques, algorithms and protocols for reducing the spectrum sensing latency while maintaining its sensing accuracy with a very low signaling overhead. Higher spectral efficiency is partly due to the extra channel reusability created by exploiting location information of the spectrum sensors through the micro-spectrum-reuse. We also formulated the optimization problem which was investigated under different system settings and proved its convexity irrespective of number of zone aggregator and base stations. As a result, a closed form solution for the optimal sensing threshold value was determined. Using simulations, the sensing accuracy and system throughput were evaluated against various network parameters to prove the efficiency of the proposed method of spectrum sensing. The simulations also demonstrated the improvement on the spectrum sensing accuracy due to the proposed method with significantly lower latency and higher system throughput comparing to the cases without zoning.

\section{ApPendix A: Proof of Lemma 1}

Proof. In this paper, we consider equiprobable hypotheses, i.e., the probability of the channel being in idle or busy states are equal. Therefore, probability of accurate channel sensing in the sensing devices is,

$$
P_{c s, i}\left(\varepsilon_{i}, T_{s, i}\right) \triangleq 1-\left\{P_{m, i}\left(\varepsilon_{i}, T_{s, i}\right)+P_{f, i}\left(\varepsilon_{i}, T_{s, i}\right)\right\}, \forall i .
$$

The cases with no sensing errors, i.e., $P_{f, i}\left(\varepsilon_{i}, T_{s, i}\right)=0$, and $P_{m, i}\left(\varepsilon_{i}, T_{s, i}\right)=0$, are referred to as perfect channel 
sensing, in such cases $P_{c s, i}\left(\varepsilon_{i}, T_{s, i}\right)=1$. However, practically $P_{c s, i}\left(\varepsilon_{i}, T_{s, i}\right) \in[0,1]$ which can be obtained by varying the operating points in ROC curve within the range, $P_{m}\left(\varepsilon_{i}, T_{s, i}\right) \leq 0.5$, and $P_{f}\left(\varepsilon_{i}, T_{s, i}\right) \leq 0.5$, which is also described in detail in $(5 c)$, (5d), and Lemmas 2-6. In the maximally inaccurate sensing case, i.e., $P_{m}\left(\varepsilon_{i}, T_{s, i}\right)=0.5$, (or $P_{d}\left(\varepsilon_{i}, T_{s, i}\right)=0.5$ ), and $P_{f}\left(\varepsilon_{i}, T_{s, i}\right)=0.5$, we get $P_{c s, i}\left(\varepsilon_{i}, T_{s, i}\right)=0$. Similarly in the perfect sensing case, i.e., $P_{m}\left(\varepsilon_{i}, T_{s, i}\right)=0$, (or $P_{d}\left(\varepsilon_{i}, T_{s, i}\right)=1$ ), and $P_{f}\left(\varepsilon_{i}, T_{s, i}\right)=0$, we get $P_{c s, i}\left(\varepsilon_{i}, T_{s, i}\right)=1$.

The logical AND rule is implemented in the ZAs, based on the sensing information collected from sensing devices. Therefore, the aggregated information is $P_{a g, i}=\prod_{i=1}^{N} P_{x, i}$, where $P_{x, i}$ is either miss detection or false alarm probability. Therefore, for any channel $i$,

$P_{c s, i}^{(Z A)}\left(\varepsilon_{i}, T_{s, i}\right)=1-\left\{\prod_{z=1}^{Z} P_{m, i}\left(\varepsilon_{i}, T_{s, i}\right)+\prod_{z=1}^{Z} P_{f, i}\left(\varepsilon_{i}, T_{s, i}\right)\right\}$.

The ZAs then make the spectrum sensing decision for each channel in its corresponding zone based on applying AND rule on the sensing decisions provided by the sensor in their corresponding zone. The obtained information is combined at the SBS such that one channel can be utilized by multiple users within the transmission range of SBS but no adjacent zones are permitted to access the same channels.

Therefore, for possible micro-spectrum-reuse, the aggregate information is $P_{a g, i}=1-\prod_{i=1}^{N}\left(1-P_{y, i}\right)$, where $P_{y, i}$ is either miss detection or false alarm probability for channel $i$. Consequently, $P_{a g, i}=0$ for $P_{y, i}=0, \forall i$ thus (24) is written as

$P_{c s, i}^{(S B S)}=1-\prod_{s=1}^{S} 1-\left[1-\prod_{z=1}^{Z} P_{m, i}\left(\varepsilon_{i}, T_{s, i}\right)-\prod_{z=1}^{Z} P_{f, i}\left(\varepsilon_{i}, T_{s, i}\right)\right]$.

Straightforward mathematical manipulations for independent decisions from ZAs result in the following

$P_{c s, i}^{(S B S)}=1-\left[\left\{1-P_{d}\left(\varepsilon_{i}, T_{s, i}\right)\right\}^{Z}+\left\{P_{f}\left(\varepsilon_{i}, T_{s, i}\right)\right\}^{Z}\right]^{S}, \forall i$

which completes the proof.

\section{APPENDIX B: LEMMA 4}

Lemma 4. If $P_{m}\left(\varepsilon_{i}, T_{s, i}\right) \leq 0.5$, and $P_{f}\left(\varepsilon_{i}, T_{s, i}\right) \leq 0.5$, then

$$
\sigma_{w}^{2} \leq \varepsilon_{i} \leq\left(1+\gamma_{i}\right) \sigma_{w}^{2} .
$$

Proof. Starting from $P_{f}\left(\varepsilon_{i}, T_{s, i}\right) \leq 0.5$ along with (1), we write:

$$
\left(\frac{\varepsilon_{i}}{\sigma_{w}^{2}}-1\right) \sqrt{T_{s, i} f_{s}} \geq \mathrm{Q}^{-1}(0.5)=0 .
$$

Since $T_{s, i} f_{s}>0$, then $\frac{\varepsilon_{i}}{\sigma_{w}^{2}}-1 \geq 0$, therefore, $\varepsilon_{i} \geq \sigma_{w}^{2}$.

Similarly, substituting (2) in $P_{m}\left(\varepsilon_{i}, T_{s, i}\right) \leq 0.5$, results in:

$$
\left(\frac{\varepsilon_{i}}{\sigma_{w}^{2}}-\gamma_{i}-1\right) \sqrt{\frac{T_{s, i} f_{s}}{2 \gamma_{i}+1}} \leq \mathrm{Q}^{-1}(0.5)=0 .
$$

Since $\frac{T_{s, i} f_{s}}{2 \gamma_{i}+1}>0$, then $\frac{\varepsilon_{i}}{\sigma_{w}^{2}}-\gamma_{i}-1 \leq 0$. Therefore, $\varepsilon_{i} \leq$ $\left(1+\gamma_{i}\right) \sigma_{w}^{2}$.

\section{APPENDIX C: LEMMA 5}

Lemma 5. For a fixed, $T_{s, i}$, and $\varepsilon_{i} \geq \sigma_{w}^{2}, P_{f}\left(\varepsilon_{i}, T_{s, i}\right)$ is a decreasing and convex function of $\varepsilon_{i}$.

Proof. Starting from (1), the first derivative of $P_{f}\left(\varepsilon_{i}, T_{s, i}\right)$ is

$$
\frac{\partial P_{f}\left(\varepsilon_{i}, T_{s, i}\right)}{\partial \varepsilon_{i}}=-\frac{\sqrt{T_{s, i} f_{s}}}{\sqrt{2 \pi} \sigma_{w}^{2}} \exp \left(-\left(\frac{\varepsilon_{i}}{\sigma_{w}^{2}}-1\right)^{2} \frac{T_{s, i} f_{s}}{2}\right),
$$

which is always negative; thus, $P_{f}\left(\varepsilon_{i}, T_{s, i}\right)$ is a decreasing function of $\varepsilon_{i}$. The second derivative of $P_{f}\left(\varepsilon_{i}, T_{s, i}\right)$ is

$$
\frac{\partial^{2} P_{f}\left(\varepsilon_{i}, T_{s, i}\right)}{\partial^{2} \varepsilon_{i}}=\frac{T_{s, i} f_{s}}{\sqrt{2 \pi} \sigma_{w}^{4}} \exp \left(-C_{1}^{2} \frac{T_{s, i} f_{s}}{2}\right)\left(\frac{\varepsilon_{i}}{\sigma_{w}^{2}}-1\right),
$$

where $C_{1}=\frac{\varepsilon_{i}}{\sigma_{w}^{2}}-1$. Since $\sigma_{w}^{2} \leq \varepsilon_{i}$, the second derivation is always positive; therefore, $P_{f}\left(\varepsilon_{i}, T_{s, i}\right)$ is a convex function of $\varepsilon_{i}$.

\section{APPENDIX D: LEMMA 6}

Lemma 6. For a fixed $T_{s, i}, \varepsilon_{i} \leq\left(1+\gamma_{i}\right) \sigma_{w}^{2}, P_{m}\left(\varepsilon_{i}, T_{s, i}\right)$ is an increasing and convex function of $\varepsilon_{i}$.

Proof. Starting from (2), the first derivative of $P_{d}\left(\varepsilon_{i}, T_{s, i}\right)$ is

$$
\frac{\partial P_{d}\left(\varepsilon_{i}, T_{s, i}\right)}{\partial \varepsilon_{i}}=-\sqrt{\frac{T_{s, i} f_{s}}{2 \pi\left(2 \gamma_{i}+1\right)}} \frac{1}{\sigma_{w}^{2}} \exp \left(-C_{2} \frac{T_{s, i} f_{s}}{2}\right),
$$

where, $C_{2}=\left(\frac{\varepsilon_{i}}{\sigma_{w}^{2}}-\gamma_{i}-1\right)^{2}$. Since $\frac{\partial P_{d}\left(\varepsilon_{i}, T_{s, i}\right)}{\partial \varepsilon_{i}}<0$, and $P_{m}\left(\varepsilon_{i}, T_{s, i}\right)=1-P_{d}\left(\varepsilon_{i}, T_{s, i}\right)$, it can be shown by substitution that $\frac{\partial P_{m}\left(\varepsilon_{i}, T_{s, i}\right)}{\partial \varepsilon_{i}}>0$. This also shows that $P_{m}\left(\varepsilon_{i}, T_{s, i}\right)$ is an increasing function of $\varepsilon_{i}$.

The second derivative of $P_{d}\left(\varepsilon_{i}, T_{s, i}\right)$ is

$$
\frac{\partial^{2} P_{d}\left(\varepsilon_{i}, T_{s, i}\right)}{\partial^{2} \varepsilon_{i}}=\sqrt{\frac{T_{s, i} f_{s}}{2 \pi\left(2 \gamma_{i}+1\right)}} \frac{1}{\sigma_{w}^{4}} \exp \left(-C_{3}\right)\left(\frac{\varepsilon_{i}}{\sigma_{w}^{2}}-\gamma_{i}-1\right) \text {, }
$$

where, $C_{3}=\left(\frac{\varepsilon_{i}}{\sigma_{w}^{2}}-\gamma_{i}-1\right)^{2} \frac{T_{s, i} f_{s}}{2}$. Here, all the terms are positive except $\frac{\varepsilon_{i}}{\sigma_{w}^{2}}-\gamma_{i}-1$. However, the inequality $\frac{\varepsilon_{i}}{\sigma_{w}^{2}}-\gamma_{i}-$ $1 \leq 0$ holds under the condition $\varepsilon_{i} \leq\left(1+\gamma_{i}\right) \sigma_{w}^{2}$. Therefore, $\frac{\partial^{2} P_{d}\left(\varepsilon_{i}, T_{s, i}\right)}{\partial^{2} \varepsilon_{i}}<0$ holds and $P_{m}\left(\varepsilon_{i}, T_{s, i}\right)=1-P_{d}\left(\varepsilon_{i}, T_{s, i}\right)$ proves that $\frac{\partial^{2} P_{m}\left(\varepsilon_{i}, T_{s, i}\right)}{\partial^{2} \varepsilon_{i}}>0$. Consequently, $P_{m}\left(\varepsilon_{i}, T_{s, i}\right)$ is an increasing and convex function of $\varepsilon_{i}$. This completes the proof.

\section{ApPendix E: ProOf of COROLlary 1}

Proof. To prove Corollary 1, the probability of channel accurate sensing in $(8 \mathrm{a})$ is shown to be concave. We start with the scenario, where $Z=S=1$.

Scenario 1: For $Z=1, S=1$, and a fixed signaling duration, $\bar{T}_{s, i}$, and frame duration, $T$, under the stated conditions in Lemma 2-Lemma 6, the probability of accurate sensing of channel $i$ is a concave function of $\varepsilon_{i}$.

In this scenario, $P_{c s, i}\left(\varepsilon_{i}\right)=1-\left(\left(1-P_{d}\left(\varepsilon_{i}\right)\right)+P_{f}\left(\varepsilon_{i}\right)\right)$. The second derivative of $P_{c s, i}$ is

$$
\frac{\partial^{2} P_{c s, i}\left(\varepsilon_{i}, \bar{T}_{s, i}\right)}{\partial^{2} \varepsilon_{i}}=-\frac{\partial^{2} P_{m}\left(\varepsilon_{i}, \bar{T}_{s, i}\right)}{\partial^{2} \varepsilon_{i}}-\frac{\partial^{2} P_{f}\left(\varepsilon_{i}, \bar{T}_{s, i}\right)}{\partial^{2} \varepsilon_{i}} .
$$


It was already shown that $\frac{\partial^{2} P_{m}\left(\varepsilon_{i}, \bar{T}_{s, i}\right)}{\partial^{2} \varepsilon_{i}}>0$, and $\frac{\partial^{2} P_{f}\left(\varepsilon_{i}, \bar{T}_{s, i}\right)}{\partial^{2} \varepsilon_{i}}>0$, therefore $\frac{\partial^{2} P_{c s, i}\left(\varepsilon_{i}, \bar{T}_{s, i}\right)}{\partial^{2} \varepsilon_{i}}<0$.

Therefore, the second derivative of $P_{c s, i}$ is negative $\forall i$, thus it is a concave function of $\varepsilon_{i}$ for which the maximum occurs at optimal detection threshold, $\varepsilon_{i}^{*}$.

Scenario 2: Following the same line of argument as in Scenario 1, the probability of accurate sensing of channel $i$ is a concave function of $\varepsilon_{i}$, for $Z=2$ and $S=1$.

In this scenario, $P_{c s, i}\left(\varepsilon_{i}\right)=1-\left(\left(1-P_{d}^{2}\left(\varepsilon_{i}\right)\right)+P_{f}^{2}\left(\varepsilon_{i}\right)\right)$. The second derivative of $P_{c s, i}$ is

$$
\frac{\partial^{2} P_{c s, i}\left(\varepsilon_{i}, \bar{T}_{s, i}\right)}{\partial^{2} \varepsilon_{i}}=-\frac{\partial^{2} P_{m}^{2}\left(\varepsilon_{i}, \bar{T}_{s, i}\right)}{\partial^{2} \varepsilon_{i}}-\frac{\partial^{2} P_{f}^{2}\left(\varepsilon_{i}, \bar{T}_{s, i}\right)}{\partial^{2} \varepsilon_{i}} .
$$

Now the second derivative of $P_{f, i}^{2}\left(\varepsilon_{i}\right)$ is

$$
\begin{aligned}
\frac{\partial^{2} P_{f}^{2}\left(\varepsilon_{i}, T_{s, i}\right)}{\partial \varepsilon_{i}^{2}}= & -2 \frac{\sqrt{T_{s, i} f_{s}}}{\sqrt{2 \pi} \sigma_{w}^{2}}\left[\exp \left(\frac{-A}{2}\right) \frac{\partial P_{f}\left(\varepsilon_{i}, T_{s, i}\right)}{\partial \varepsilon_{i}}\right. \\
& \left.+\bar{P}_{f} \frac{\partial}{\partial \varepsilon_{i}}\left(\exp \left(\frac{-A}{2}\right)\right)\right],
\end{aligned}
$$

where $A=T_{s, i} f_{s}\left(\frac{\varepsilon_{i}}{\sigma_{w}^{2}}-1\right)^{2}$. According to Lemma 5, $\frac{\partial P_{f}\left(\varepsilon_{i}, T_{s, i}\right)}{\partial \varepsilon_{i}}<0$; we further notice that for $\bar{P}_{f}>0$ and any $x \neq 0, \frac{\partial e^{-x}}{\partial x}<0$. Using (28), we conclude that $\frac{\partial^{2} P_{f}^{2}\left(\varepsilon_{i}, T_{s, i}\right)}{\partial \varepsilon_{i}^{2}}>0$.

Following the same line of argument as in (28), it is straightforward to show that $\frac{\partial^{2} P_{d}^{2}\left(\varepsilon_{i}, T_{s, i}\right)}{\partial \varepsilon_{i}^{2}}>0$. Substituting $P_{d, i}^{2}\left(\varepsilon_{i}\right)=\left(1-P_{m, i}\left(\varepsilon_{i}\right)\right)^{2}$, we then get:

$\frac{\partial^{2} P_{d}^{2}\left(\varepsilon_{i}, T_{s, i}\right)}{\partial \varepsilon_{i}^{2}}=\left(-2 \frac{\partial^{2} P_{m}\left(\varepsilon_{i}, T_{s, i}\right)}{\partial \varepsilon_{i}^{2}}+\frac{\partial^{2} P_{m}^{2}\left(\varepsilon_{i}, T_{s, i}\right)}{\partial \varepsilon_{i}^{2}}\right)>0$.

According to Lemma 6, we also have $\frac{\partial^{2} P_{m}\left(\varepsilon_{i}, T_{s, i}\right)}{\partial \varepsilon_{i}^{2}}>0$, therefore we conclude $\frac{\partial^{2} P_{m}^{2}\left(\varepsilon_{i}, T_{s, i}\right)}{\partial \varepsilon^{2}}>0$.

From (28) and noting that the positive sum of two concave functions is also a concave function, one can conclude that $\frac{\partial^{2} P_{c s, i}\left(\varepsilon_{i}, T_{s, i}\right)}{\partial \varepsilon^{2}}<0$. It means the probability of accurate sensing is a concave and decreasing function of $\varepsilon_{i}$.

Scenario 3: Similar to Scenario 1 and 2, the probability of accurate sensing of channel $i$ is concave for $Z=1$ and $S=2$. In this case, the probability of accurate sensing is calculated as below.

$$
\begin{aligned}
P_{c s, i}\left(\varepsilon_{i}\right) & =1-\left[P_{m}\left(\varepsilon_{i}\right)+P_{f}\left(\varepsilon_{i}\right)\right]^{2} \\
& =1-P_{m}^{2}\left(\varepsilon_{1}\right)-2 P_{m}\left(\varepsilon_{1}\right) P_{f}\left(\varepsilon_{1}\right)-P_{f}^{2}\left(\varepsilon_{1}\right) .
\end{aligned}
$$

The second order derivative of (29) is

$$
\begin{aligned}
\frac{\partial^{2} P_{c s, i}^{2}\left(\varepsilon_{i}\right)}{\partial \varepsilon_{i}^{2}}= & -\frac{\partial^{2} P_{m}^{2}\left(\varepsilon_{i}\right)}{\partial \varepsilon_{i}^{2}}-2 \bar{P}_{m} \frac{\partial^{2} P_{f}\left(\varepsilon_{i}\right)}{\partial \varepsilon_{i}^{2}} \\
& -2 \bar{P}_{f} \frac{\partial^{2} P_{m}\left(\varepsilon_{i}\right)}{\partial \varepsilon_{i}^{2}}-\frac{\partial^{2} P_{f}^{2}\left(\varepsilon_{i}\right)}{\partial \varepsilon_{i}^{2}} .
\end{aligned}
$$

For a fixed signaling duration $\bar{T}_{s, i}$, we already showed that $\frac{\partial^{2} P_{f}\left(\varepsilon_{i}\right)}{\partial \varepsilon_{i}^{2}}>0$ in Lemma 5, $\frac{\partial^{2} P_{m}\left(\varepsilon_{i}\right)}{\partial \varepsilon_{i}^{2}}>0$ in Lemma 6, and $\frac{\partial^{2} P_{m}^{2}\left(\varepsilon_{i}\right)}{\partial \varepsilon_{i}^{2}}>0$, and $\frac{\partial^{2} P_{f}^{2}\left(\varepsilon_{i}\right)}{\partial \varepsilon_{i}^{2}}>0$ in Scenario 2. Moreover, we note that the maximum acceptable probability of miss detection, and false alarm are bounded as $0 \leq \bar{P}_{f} \leq 0.5$, and $0 \leq \bar{P}_{m} \leq 0.5$, respectively. Therefore, we can conclude that $\frac{\partial^{2} P_{c s, i}^{2}\left(\varepsilon_{i}\right)}{\partial \varepsilon_{i}^{2}}<0$ and is concave function of $\varepsilon_{i}$, and the maximum value occurs at $\varepsilon_{i}=\varepsilon_{i}^{*}$ which has been obtained in Section IV-D.

Scenario 4: Following the same line of argument as in Scenarios 1-3, the probability of accurate sensing of channel $i$ is a concave function of $\varepsilon_{i}$ for $Z=1, S=3$, as well as $Z=3, S=1$.

We then argue that the function is concave for the combination of ZAs, i.e., $n_{1}=2$ and $n_{2}=n_{1}+1$ and SBSs, i.e., $n_{3}=2$ and $n_{4}=n_{3}+1$ and it also holds for $n_{1}=1$ and $n_{3}=1$. It means that it must be also true for any possible combination of natural numbers of ZAs and SBSs according to the principal of mathematical induction.

\section{REFERENCES}

[1] S. Haykin, "Cognitive radio: brain-empowered wireless communications," IEEE J. Sel. Areas Commun., vol. 23, no. 2, pp. 201-220, 2005.

[2] M. Khoshkholgh, K. Navaie, and H. Yanikomeroglu, "Access strategies for spectrum sharing in fading environment: Overlay, underlay, and mixed," IEEE Trans. Mobile Comput., vol. 9, no. 12, pp. 1780-1793, 2010.

[3] Y.-C. Liang, Y. Zeng, E. Peh, and A. T. Hoang, "Sensing-throughput tradeoff for cognitive radio networks," IEEE Trans. Wireless Commun., vol. 7, no. 4, pp. 1326-1337, 2008.

[4] S. Stotas and A. Nallanathan, "Optimal sensing time and power allocation in multiband cognitive radio networks," IEEE Trans. Commun., vol. 59, no. 1, pp. 226-235, 2011.

[5] M. Khoshkholgh, K. Navaie, and H. Yanikomeroglu, "Optimal design of the spectrum sensing parameters in the overlay spectrum sharing," IEEE Trans. Mobile Comput., vol. PP, no. 99, pp. 1-1, 2013.

[6] A. Ghasemi and E. Sousa, "Collaborative spectrum sensing for opportunistic access in fading environments," in New Frontiers in Dynamic Spectrum Access Networks, 2005. DySPAN 2005. 2005 First IEEE International Symposium on, Nov 2005, pp. 131-136.

[7] H. Zhuang, Z. Luo, J. Zhang, and Y. Li, "Cooperative sensor solution to enhancing the performance of spectrum sensing," in Communications and Networking in China (CHINACOM), 2010 5th International ICST Conf. on, Aug 2010, pp. 1-6.

[8] Y. Abdi and T. Ristaniemi, "Joint local quantization and linear cooperation in spectrum sensing for cognitive radio networks," IEEE Trans. Signal Process., vol. 62, no. 17, pp. 4349-4362, Sept 2014.

[9] E. Peh, Y.-C. Liang, Y. L. Guan, and Y. Zeng, "Cooperative spectrum sensing in cognitive radio networks with weighted decision fusion schemes," IEEE Trans. Wireless Commun., vol. 9, no. 12, pp. 38383847, December 2010.

[10] D. Xue, E. Ekici, and M. C. Vuran, "Cooperative spectrum sensing in cognitive radio networks using multidimensional correlations," IEEE Trans. Wireless Commun., vol. 13, no. 4, pp. 1832-1843, April 2014.

[11] I. F. Akyildiz, B. F. Lo, and R. Balakrishnan, "Cooperative spectrum sensing in cognitive radio networks: A survey," Phys. Commun., vol. 4, no. 1, pp. 40-62, Mar. 2011. [Online]. Available: http://dx.doi.org/10.1016/j.phycom.2010.12.003

[12] H. Yu, W. Tang, and S. Li, "Optimization of multiple-channel cooperative spectrum sensing with data fusion rule in cognitive radio networks," Journal of Electronics (China), vol. 29, no. 6, pp. 515-522, 2012.

[13] W. Lin, Y. Wang, and W. Ni, "Cluster-based cooperative spectrum sensing in two-layer hierarchical cognitive radio networks," in Global Comm. Conf. (GLOBECOM), 2013 IEEE, Dec 2013, pp. 1082-1087.

[14] W. Zhang, Y. Yang, and C. K. Yeo, "Cluster-based cooperative spectrum sensing assignment strategy for heterogeneous cognitive radio network," IEEE Trans. Veh. Technol., vol. 64, no. 6, pp. 2637-2647, June 2015.

[15] C. G. Tsinos and K. Berberidis, "Decentralized adaptive eigenvaluebased spectrum sensing for multiantenna cognitive radio systems," vol. 14 , no. 3, pp. 1703-1715, March 2015. 
[16] Y. Han, S. H. Ting, and A. Pandharipande, "Cooperative spectrum sharing protocol with secondary user selection," IEEE Trans. Wireless Commun., vol. 9, no. 9, pp. 2914-2923, September 2010.

[17] I. Nevat, G. W. Peters, and I. B. Collings, "Location-aware cooperative spectrum sensing via gaussian processes," in Communications Theory Workshop (AusCTW), 2012 Australian, Jan 2012, pp. 19-24.

[18] J. Lai, E. Dutkiewicz, R. Liu, and R. Vesilo, "Opportunistic spectrum access with two channel sensing in cognitive radio networks," IEEE Trans. Mobile Comput., vol. PP, no. 99, pp. 1-1, 2013.

[19] M. Monemian and M. Mahdavi, "Analysis of a new energy-based sensor selection method for cooperative spectrum sensing in cognitive radio networks," IEEE Sensors J., vol. 14, no. 9, pp. 3021-3032, Sept 2014.

[20] Y. Selen, H. Tullberg, and J. Kronander, "Sensor selection for cooperative spectrum sensing," in New Frontiers in Dynamic Spectrum Access Networks, DySPAN 2008. 3rd IEEE Symposium on, Oct 2008, pp. 1-11.

[21] Y. Li, K. K. Chai, Y. Chen, and J. Loo, "Duty cycle control with joint optimisation of delay and energy efficiency for capillary machineto-machine networks in 5G communication system," Transactions on Emerging Telecomm. Tech., vol. 26, no. 1, pp. 56-69, 2015. [Online]. Available: http://dx.doi.org/10.1002/ett.2891

[22] B. Mercier, V. Fodor, R. Thobaben, M. Skoglund, V. Koivunen, S. Lindfors, J. Ryynänen, E. G. Larsson, C. PetriolI, G. Bongiovanni et al., "Sensor networks for cognitive radio: Theory and system design," ICT mobile summit, 2008.

[23] O. Grondalen, M. Lahteenoja, and P. Gronsund, "Evaluation of business cases for a cognitive radio network based on wireless sensor network," in New Frontiers in Dynamic Spectrum Access Networks (DySPAN), 2011 IEEE Symposium on, May 2011, pp. 242-253.

[24] N. Hasan, W. Ejaz, S. Lee, and H. Kim, "Knapsack-based energyefficient node selection scheme for cooperative spectrum sensing in cognitive radio sensor networks," Commun., IET, vol. 6, no. 17, pp. 2998-3005, November 2012.

[25] S. Maleki, A. Pandharipande, and G. Leus, "Energy-efficient distributed spectrum sensing for cognitive sensor networks," Sensors Journal, IEEE, vol. 11, no. 3, pp. 565-573, 2011.

[26] K. Letaief and W. Zhang, "Cooperative communications for cognitive radio networks," Proceedings of the IEEE, vol. 97, no. 5, pp. 878-893, May 2009.

[27] H. Cramér, Mathematical methods of statistics. Princeton university press, 1999, vol. 9.

[28] S. Atapattu, C. Tellambura, and H. Jiang, "Analysis of area under the ROC curve of energy detection," IEEE Trans. Wireless Commun., vol. 9, no. 3, pp. 1216-1225, March 2010.

[29] “IEEE 802.22 WRAN WG," http://www.ieee802.org/22/, 2012.

[30] A. Singh, M. Bhatnagar, and R. Mallik, "Cooperative spectrum sensing in multiple antenna based cognitive radio network using an improved energy detector,' IEEE Commun. Lett., vol. 16, no. 1, pp. 64-67, 2012.

[31] P. Paysarvi-Hoseini and N. Beaulieu, "Optimal wideband spectrum sensing framework for cognitive radio systems," IEEE Trans. Signal Process., vol. 59, no. 3, pp. 1170-1182, March 2011.

[32] R. Fan and H. Jiang, "Optimal multi-channel cooperative sensing in cognitive radio networks," IEEE Trans. Wireless Commun., vol. 9, no. 3, pp. 1128-1138, March 2010.

[33] S. P. Boyd and L. Vandenberghe, Convex optimization. Cambridge university press, 2004.

[34] W. Wang, B. Kasiri, J. Cai, and A. S. Alfa, "Channel assignment schemes for cooperative spectrum sensing in multi-channel cognitive radio networks," Wireless Communications and Mobile Computing, vol. 15, no. 10, pp. 1471-1484, 2015. 